\title{
Dynamic coupling in Cooker's sloshing experiment with baffles.
}

\author{
M. R. Turner ${ }^{1}$, T. J. Bridges \& H. Alemi Ardakani \\ Department of Mathematics, University of Surrey, Guildford GU2 7XH, U.K.
}

\begin{abstract}
This paper investigates the dynamic coupling between fluid sloshing and the motion of the vessel containing the fluid, for the case when the vessel is partitioned using non-porous baffles. The vessel is modelled using Cooker's sloshing configuration [M. J. Cooker, Wave Motion 20, 385-395 (1994)]. Cooker's configuration is extended to include $n-1$ non-porous baffles which divide the vessel into $n$ separate fluid compartments each with a characteristic length scale. The problem is analysed for arbitrary fill depth in each compartment, and it is found that a multitude of resonance situations can occur in the system, from $1: 1$ resonances to $(n+1)$-fold $1: 1: \cdots: 1$ resonances, as well as $\ell: m: \cdots: n$ for natural numbers $\ell, m, n$, depending upon the system parameter values. The conventional wisdom is that the principle role of baffles is to damp the fluid motion. Our results show that in fact without special consideration, the baffles can lead to enhancement of the fluid motion through resonance.
\end{abstract}

Key words: sloshing, baffles, $(n+1)$-fold resonance, finite depth, coupling

\section{Introduction}

The sloshing of fluid in a moving container can lead to the destabilization of the vessel. One of the first strategies for minimizing sloshing was to introduce baffles. The space industry was an early example where a wide range of baffling was tested (e.g. (1), (2), (3)). Indeed careful placement of baffles can be highly successful in reducing fluid motion and fluid loading. This is accomplished first and foremost by simply blocking the fluid motion. A second more subtle effect of baffles is to change the natural frequency of the fluid (e.g. see $\S 4.7 .2$ of (4)). Hence the introduction of baffles can be used to tune the principle natural frequency away from ambient frequencies.

It is this secondary effect that we investigate in this paper. In addition to looking at the effect of baffles on natural frequencies, we add in vessel motion. Coupling the fluid motion with the vessel motion brings in an additional potential source of resonance. The simplest vessel motion is to attach the vessel at a single point and allow rotary motion; that is, a pendulum model

$\overline{1}$ Corresponding author: email: M.Turner@surrey.ac.uk. 
for the vessel. Such a model, without baffles, was first considered by Moiseyev \& Rumyantsev (5) (see $§ 5-4$ in (5)). However, it would be difficult to construct an experiment with this configuration. Cooker (6) discovered that if two suspension points were used, a robust and simple experiment could be constructed. This configuration is one of the simplest experiments which demonstrates the dynamic coupling between a sloshing fluid and the motion of the vessel holding the fluid. It is the Cooker configuration that is the model used in this paper for the vessel motion. However, the principles used (resonance between vessel and fluid, and resonance between compartments) are general and will be applicable to other configurations.

The first study linking dynamic coupling, baffles and resonance was the experiments of Weidman $(7 ; 8)$. He extended the shallow water theory of Cooker (6) to a multi-compartment rectangular vessel and found that the frequency of the lowest frequency solution reduced as more compartments were filled. Weidman also obtained experimental results for this configuration for various cable lengths. In this paper the results of Weidman are extended to the case of arbitrary depth. The overall aim is to study the resonance structure of the dynamic coupling between the vessel motion and the fluid motion in a baffled rectangular container, with arbitrary fill depth in each compartment. In addition to the fundamental interest, understanding the coupling mechanism in this problem has great practical implications in areas such as terrestrial fluid transportation, maritime and space transportation, storage tanks under earthquake excitation and industrial applications.

The experiment devised by Cooker (6) consists of a rectangular vessel containing fluid suspended by two cables which can rotate in a vertical plane. A schematic diagram of the experimental set up is shown in figure 1 . The vessel has length $L$, width $W$, height $d$ and is partially

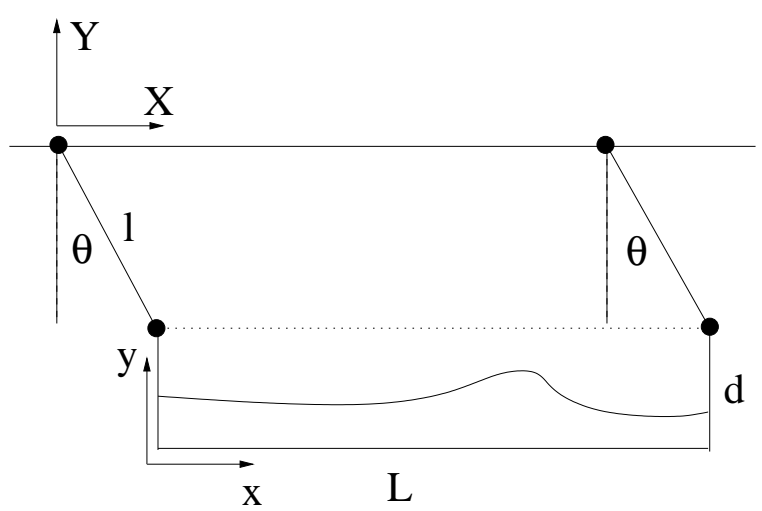

Fig. 1. A schematic illustration of Cooker's sloshing experiment.

filled with a fluid of mean height $H_{0}<d$ and density $\rho$. The suspending cables are of length $l$ and they make an angle $\theta$ with the vertical axis. The vessel is released with initial values of $\theta$ and $d \theta / d t$, and the vessel swings in the vertical plane causing the fluid to slosh back and forth along the vessel, during which time the base of the vessel remains horizontal.

Cooker (6) obtained good theoretical agreement with his experimental apparatus using a linear harmonic equation for the vessel motion and a linear shallow water model for the fluid motion. The theoretical results showed that the presence of the sloshing fluid changed the natural oscillation frequency of the vessel motion. This theory was extended to a nonlinear shallow water model, still with a linear vessel equation, by Alemi Ardakani \& Bridges (9), who developed a numerical scheme for solving the coupled problem which conserved the total energy in the system, as well as giving the correct energy partition between the fluid and the vessel. In his analysis, Cooker (6) found a curious resonance where the second mode of the coupled problem 
appeared to resonate with the natural frequency of the vessel. This behaviour could not be explained, as the resonant solution did not satisfy the dispersion relation. This non-resonant behaviour was supported by $\mathrm{Yu}(10)$ who extended the linear vessel motion problem to include a fluid of finite depth, but could not find a mechanism for continued energy input to support a resonant behaviour. This problem was resolved by Alemi Ardakani et al. (11; 12) who carefully derived the dispersion relation in the finite depth problem and highlighted the internal $1: 1$ resonance where the natural frequency of the dry vessel resonates with one of the fluid modes. The key feature of the solution is that the dispersion relation is the product of two functions

$$
\Delta(\omega)=P(\omega) D(\omega),
$$

where $\omega$ is the frequency of the motion and $D(\omega)$ is the dispersion relation in the works of Cooker (6) or $\mathrm{Yu}(10)$. The roots of $D(\omega)=0$ correspond to modes which couple the antisymmetric fluid modes to the vessel motion, while the roots of $P(\omega)=0$ are associated with the symmetric fluid modes. Natural frequencies satisfy $\Delta(\omega)=0$, but when both $P(\omega)=D(\omega)=0$ with both $P^{\prime}(\omega)$ and $D^{\prime}(\omega)$ non-zero, a $1: 1$ resonance occurs (or a $1: 1$ semisimple resonance) where both modes couple together $(13 ; 14)$.

The natural frequencies for the dynamic coupling between Cooker's vessel configuration and an unbaffled tank were computed in by Alemi Ardakani et al. (11). In this paper, the finite-depth theory is extended to investigate the coupled sloshing problem when $n-1$ non-porous baffles are inserted into the vessel, creating $n$ separate fluid compartments that can be filled with fluids of different densities and heights. The consequence of the baffles is to create a system where $D(\omega)$ now couples the anti-symmetric modes of all the compartments, and $P(\omega)$ is the product of contributions from symmetric modes in each compartment. Therefore, this system can support many different resonant behaviours, from $1: 1$ resonances, $1: 1: 1$ resonances up to $(n+1)$-fold $1: 1: \cdots: 1$ resonances. This is because now $P(\omega)=0$ can be accompanied by $P^{\prime}(\omega)=\cdots=P^{(n-1)}(\omega)=0$. If this combination occurs when $D(\omega)=0$ the $(n+1)$-fold $1: 1: \cdots: 1$ resonance is observable. The effect of the model parameters on the occurring resonances as well as the lowest frequency sloshing mode are investigated numerically in this article.

At this stage it is worth noting that the coupled problem considered here is similar to that of tuned liquid dampers (TLDs) $(15 ; 16)$. A TLD consists of a vessel partially filled with fluid, but it is now constrained to move only in the horizontal plane with a restoring force given by a mass-spring-damper model. Alemi Ardakani et al. (11) showed that in the linear regime the two systems are equivalent, but their nonlinear structure is different due to vertical displacements of the vessel occurring in Cooker's experiment. Ikeda (17) studied two TLDs in series, the linear regime of which is equivalent to the linear regime of the two-compartment case in this study. Ikeda found parameter values in the nonlinear regime where out-of-phase sloshing occurs between two compartments. Although Ikeda (17) only considered two compartments the TLD configuration could be extended to $n$ compartments as here, leading to a potential $(n+1)-$ fold resonance in TLDs.

The $1: 1$ resonance has been observed in other physical systems, the most well known example is probably the Faraday experiment in a vessel with a square cross section ((18); see also $§ 9.2 .3$ of Faltinsen \& Timokha (4)). These 1: 1 resonances are of practical interest as the nonlinear problem close to a 1:1 resonance has multiple bifurcations of periodic solutions which, can cause chaotic dynamics which are more dramatic than forcing close to simple frequencies (13). Turner \& Bridges (19) identified a heteroclinic orbit close to the $1: 1$ resonance in Cooker's experiment, for one particular fluid height $H_{0}$, which allowed an energy transfer between the purely symmetric fluid modes and the purely anti-symmetric fluid modes. The energy transfer 
in this case produced erratic behaviour in the system where the oscillating vessel would come to rest as the vessel energy was transferred to the symmetric fluid modes, before oscillating again once the energy was transferred back to the anti-symmetric fluid modes and hence the vessel. A similar behaviour was observed by Struble \& Heinbockel (20) in their simplified version of Cooker's experiment where the vessel was replaced by an elastic beam. In this case the internal resonance was a $1: 2$ resonance. Other systems with $1: 1$ resonances include orthogonal pendulums (21) and the Hénon-Heiler system in celestial mechanics (22). The trifold $1: 1: 1$ and four-fold $1: 1: 1: 1$ resonances are much less common, but examples for both have been observed in chemistry. The $1: 1: 1$ resonance was found in vibrations of tetrahedral molecules $(23 ; 24)$ and the $1: 1: 1: 1$ resonance in hydrogen atoms in crossed electric and magnetic fields (25). Resonances other than the $1: 1,1: 1: 1$ and $1: 1: 1: 1$ resonances have been seen in experiments and physical problems before, such as the $1: 1: 2$ internal resonance in an elastic conical pendulum (26) or a $2: 1: 1: 1$ internal resonance in a wake oscillator model for a spherical pendulum (27) to name a couple. Such resonances are also possible in our experimental setup. To our knowledge the system analysed in this paper indicates the first time the $(n+1)$-fold $1: 1: \cdots: 1$ resonance has been seen in a problem with a real practical application for $n \geq 4$.

The current paper is laid out as follows. In section 2 we formulate the nonlinear, finite depth fluid equations, and the nonlinear vessel equation, while in $\S 3$ we consider solutions to the linear form of this problem. The dispersion relation for the $n$ fluid problem is investigated and its resonance properties are considered in $\S 4$ for the case $n=2$ as well as the general case. This section also contains numerical solutions of the dispersion relation for the lowest frequency solution, which is likely to be the solution observed in experiments. A brief discussion of the shallow water limit can be found in $\S 5$, and our concluding remarks are given in $\S 6$.

\section{Governing equations}

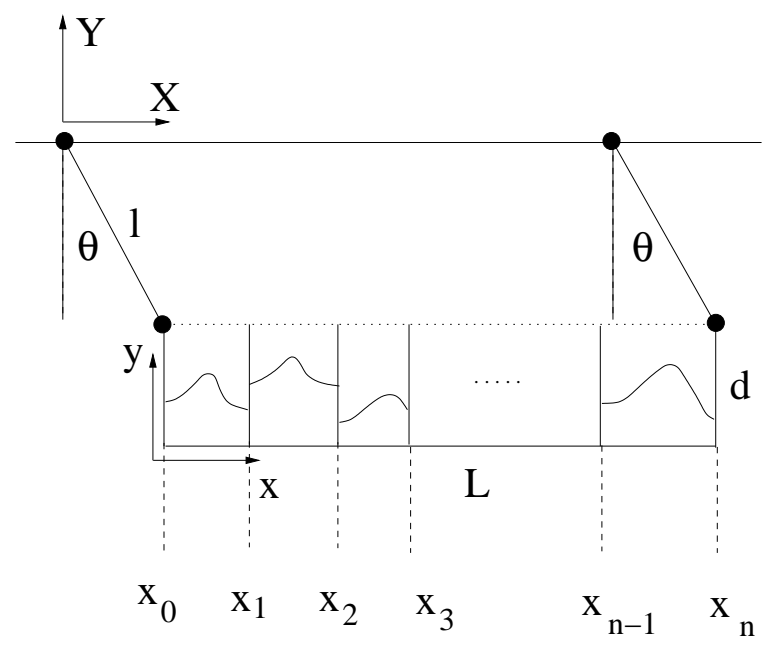

Fig. 2. A schematic illustration of Cooker's sloshing experiment, with the introduction of $n-1$ baffles at $x=x_{i}$ for $i=1, \ldots, n-1$.

A schematic illustration of the sloshing system under investigation in this article is shown in figure 2. The fixed coordinate system $(X, Y)$ has its origin at the left most fixed point of the suspension cable, and the coordinates $(x, y)$ are attached to the moving vessel. These two 
coordinate systems are related by

$$
X=x+q_{1}, \quad Y=y+q_{2}-d,
$$

where

$$
q_{1}(t)=l \sin \theta(t), \quad q_{2}(t)=-l \cos \theta(t),
$$

and the motion is constrained such that $q_{1}^{2}+q_{2}^{2}=l^{2}$. The fluid compartments are labelled from left to right, and in each of the $n$ compartments, the fluid occupies

$$
0 \leq y \leq h^{(i)}(x, t) \quad \text { and } \quad x_{i-1} \leq x \leq x_{i}
$$

for $i=1,2, \ldots, n$, where $x_{j}<x_{k}$ for $j<k$ with $x_{0}=0$ and $x_{n}=L$. The length of each compartment is then $L_{i}=x_{i}-x_{i-1}$. In each compartment we assume the fluid has density $\rho_{i}$, and is irrotational and incompressible so its motion can be represented by the velocity potential $\phi^{(i)}(x, y, t)$.

Alemi Ardakani et al. (11) derived the nonlinear equations of motion for a single compartment vessel which can be easily extended to a vessel with $n$ compartments. In each compartment the velocity potential satisfies

$$
\begin{aligned}
& \phi_{x x}^{(i)}+\phi_{y y}^{(i)}=0, \text { in } 0 \leq y \leq h^{(i)}(x, t), x_{i-1} \leq x \leq x_{i}, \\
& \phi_{t}^{(i)}+\frac{1}{2}\left[\left(\phi_{x}^{(i)}\right)^{2}+\left(\phi_{y}^{(i)}\right)^{2}\right]-\dot{q}_{1} \phi_{x}^{(i)}-\dot{q}_{2} \phi_{y}^{(i)}+g h^{(i)}=0, \text { on } y=h^{(i)}(x, t), \\
& h_{t}^{(i)}+\left(\phi_{x}^{(i)}-\dot{q}_{1}\right) h_{x}^{(i)}=\phi_{y}^{(i)}-\dot{q}_{2}, \text { on } y=h^{(i)}(x, t), \\
& \phi_{y}^{(i)}=\dot{q}_{2} \text { on } y=0, \\
& \phi_{x}^{(i)}=\dot{q}_{1} \text { on } x=x_{i-1}, x_{i},
\end{aligned}
$$

for $i=1, \ldots, n$, where the subscripts denote partial derivatives and dots denote full derivatives with respect to time. Equations (2.5) and (2.6) are the dynamic and kinematic free surface conditions on $y=h^{(i)}(x, t)$ and (2.7) and (2.8) are the no penetration conditions on the bottom and side walls of the vessel. The motion of the fluid is coupled to the vessel motion via a nonlinear forced pendulum equation

$$
m_{v} \ddot{\theta}+\frac{g M}{l} \sin \theta=-\frac{1}{l} \sum_{i=1}^{n}\left(\dot{\sigma}_{i} \cos \theta+\dot{\tau}_{i} \sin \theta\right),
$$

where $m_{v}$ is the vessel mass, $M=m_{v}+\sum_{i=1}^{n} m_{f}^{(i)}$ is the total mass and

$$
\sigma_{i}=W \int_{x_{i-1}}^{x_{i}} \int_{0}^{h^{(i)}} \rho_{i} \phi_{x}^{(i)} d y d x \quad \tau_{i}=W \int_{x_{i-1}}^{x_{i}} \int_{0}^{h^{(i)}} \rho_{i} \phi_{y}^{(i)} d y d x
$$

This completes the derivation of the nonlinear equations. 


\section{Solution to the linearized problem}

Here we linearize (2.4)-(2.9) about the quiescent solution, where $\theta=0, h^{(i)}=H_{i}$ and $\phi^{(i)}=$ $f^{(i)}(t)$ for all $i$. Here each $H_{i}$ is a constant and each $f^{(i)}(t)$ is arbitrary and doesn't affect the overall solution. To leading order, the forcing terms are $\dot{q}_{1} \sim l \dot{\theta}$ and $\dot{q}_{2} \sim 0$, so the linear form of the governing nonlinear fluid equations in $\S 2$ reduce to

$$
\begin{aligned}
& \phi_{x x}^{(i)}+\phi_{y y}^{(i)}=0, \text { in } 0 \leq y \leq H_{i}, x_{i-1} \leq x \leq x_{i}, \\
& \phi_{t}^{(i)}+g h^{(i)}=0, \text { and } h_{t}^{(i)}=\phi_{y}^{(i)} \Longrightarrow \phi_{t t}^{(i)}+g \phi_{y}^{(i)}=0 \text { on } y=H_{i}, \\
& \phi_{y}^{(i)}=0 \text { on } y=0, \\
& \phi_{x}^{(i)}=l \dot{\theta} \text { on } x=x_{i-1}, x_{i},
\end{aligned}
$$

for each fluid compartment and

$$
m_{v} l \ddot{\theta}+W \sum_{i=1}^{n} \int_{x_{i-1}}^{x_{i}} \int_{0}^{H_{i}} \rho_{i} \phi_{x t}^{(i)} d y d x+g M \theta=0
$$

for the vessel.

This system of linear equations is solved by searching for time periodic solutions with some undetermined frequency $\omega$ and then expanding the spatial part of $\phi$ as an infinite sum of vertical eigenmodes $(28 ; 10)$. This approach is similar to that used in Alemi Ardakani et al. (11) and leads to a dispersion relation of the form

$$
\Delta_{n}(\omega)=P_{n}(\omega) D_{n}(\omega),
$$

where

$$
\begin{aligned}
P_{n}(\omega)= & \prod_{i=1}^{n} \sin \left[\alpha_{0}^{(i)}\right] \\
D_{n}(\omega)= & \left(-\left(M g-m_{v} l \omega^{2}\right)+2 l \omega^{2} W \sum_{i=1}^{n} \rho_{i} H_{i} \frac{\left(c_{0}^{(i)}\right)^{2}}{k_{0}^{(i)}} \tan \left[\alpha_{0}^{(i)}\right]\right. \\
& \left.+2 l \omega^{2} W \sum_{i=1}^{n} \rho_{i} H_{i} \sum_{m=1}^{\infty} \frac{\left(c_{m}^{(i)}\right)^{2}}{k_{m}^{(i)}} \tanh \left[\alpha_{m}^{(i)}\right]\right) \prod_{i=1}^{n} \cos \left[\alpha_{0}^{(i)}\right]
\end{aligned}
$$

and the form of the $c_{j}^{(i)}$, s and $k_{j}^{(i)}$ 's are given in (A.9) and (A.8) respectively and $\alpha_{j}^{(i)}=\frac{1}{2} k_{j}^{(i)} L_{i}$. A full derivation of the dispersion relation is given in appendix A. A derivation of the dispersion relation can also be obtained from the general expressions for the added mass coefficients due to the sloshing fluid ((4), Chapter 5). However, the derivation presented in appendix A is designed to be accessible to the general reader who might not familiar with the added mass coefficient approach.

In (3.8) we have divided out the quantity $\prod_{i=1}^{n} 2^{n} k_{0}^{(i)}$ which appears in (A.15), because it is non-zero as we assume that $\omega \neq 0$. The dispersion relation, $\Delta_{n}=0$, is an implicit equation for 
$\omega$ which depends on the parameters $m_{v}, l, L, W$ for the vessel and $x_{i}, \rho_{i}, H_{i}$ for $i=1, \ldots, n$ for the fluid compartments. It also has a form similar to that of $(1.1)$ where $P_{n}(\omega)$ consists of contributions from symmetric sloshing modes and $D_{n}(\omega)$ consists of anti-symmetric modes coupled to the vessel motion.

\subsection{Numerical evaluation of the dispersion relation}

The roots of $\Delta_{n}(\omega)=0$ which correspond to $P_{n}(\omega)=0$ can be determined analytically, but the roots which correspond to $D_{n}(\omega)=0$ need to be evaluated numerically. We assume that all the roots of $D_{n}(\omega)=0$ are real, and to determine the eigenvalues $\omega$ for which $D_{n}(\omega)=0$, we plot $D_{n}$ as a function of $\omega$ and calculate where this function crosses the $\omega$-axis. These roots can then be refined via Newton's method if necessary. At each value of $\omega$, the values of $k_{m}^{(i)}$ need to be determined for $m=0, . ., M$ for each $i=1, \ldots, n$, where $M$ is a large enough integer such that $D_{n}(\omega)$ is independent of $M$. One approach to calculating the $k_{m}^{(i)}$ values is to directly solve (A.8) using Newton iterations or to solve the transcendental equation associated with the exact characteristic function (see appendix B of (11)). The main problem with this approach is the difficulty selecting the initial guess for each $k_{m}^{(i)}$ so that firstly, the iterative method converges and secondly, no values of $k_{m}^{(i)}$ are missed.

These problems can be overcome by solving the vertical eigenvalue problem given in the appendix of Alemi Ardakani et al. (11) directly, using Chebyshev spectral collocation methods (29). In this approach we transform the domain from $y \in\left[0, H_{i}\right]$ to $\bar{y} \in[-1,1]$ and assume that

$$
\psi(\bar{y})=\sum_{j=0}^{N_{C}} a_{j} T_{j}(\bar{y}),
$$

where $T_{j}(\bar{y})$ are Chebyshev polynomials, and $a_{i}$ are undetermined parameters. Evaluating this expression at the $N_{C}-1$ collocation points

$$
\bar{y}_{j}=\cos \left(\frac{j \pi}{N_{C}}\right), \quad j=1, \ldots, N_{C}-1
$$

and at the two boundaries $\bar{y}=-1$ and $\bar{y}=1$, gives $N_{C}+1$ algebraic equations for the eigenvalues $\lambda$, reducing the equation for the vertical eigenfunctions to the matrix eigenvalue problem

$$
\mathbf{A} \Psi=\lambda \Psi, \quad \Psi \in \mathbb{R}^{N_{C}+1} .
$$

The main benefit of this approach is that all the eigenvalues for the chosen approximation, and hence the values of $k_{m}^{(i)}$, are calculated in one go, without any being missed. By choosing $N_{C}$ large enough, the first $M$ modes can be calculated to the desired accuracy.

The results to be presented in $\S 4$ use $M=10$ evanescent modes, and so using $N_{C}=50$ in the spectral collocation approach above is sufficient to calculate the values of $k_{m}^{(i)}$ to 12 significant figures. These values were checked against values calculated via an iterative solution of (A.8). $\mathrm{Yu}$ (10) found that 5 evanescent modes for the finite depth unbaffled problem was sufficient to determine the lowest frequency solutions, however as the number of baffles is increased in the vessel, the fluid depth in each compartment can become large compared to the compartment length, so we use 10 modes to be cautious.

An example of $\Delta_{1}(\omega)$ is plotted in figure 3. Once a root of $\Delta_{1}(\omega)=0$ is found, the values of $k_{m}^{(1)}$ 


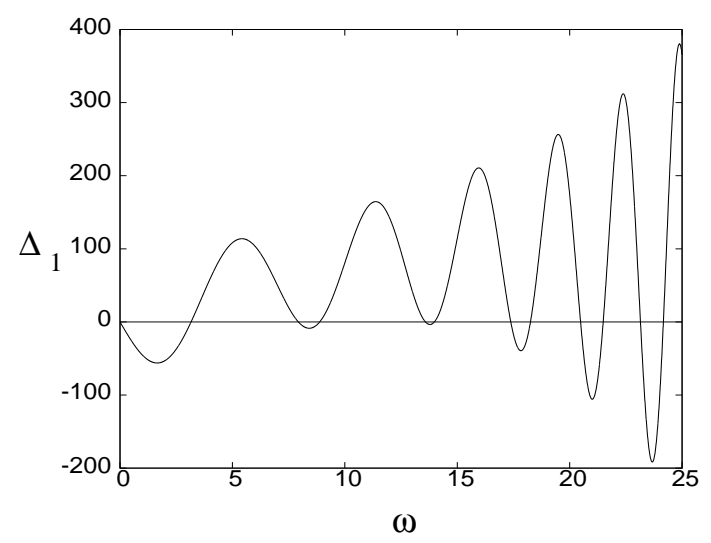

Fig. 3. Plot of $\Delta_{1}(\omega)$ with $H_{1}=0.05 \mathrm{~m}, l=0.5 \mathrm{~m}$ and the other parameters given in table 1 .

Table 1

\begin{tabular}{ll}
\hline Parameter & $\begin{array}{l}\text { Value or } \\
\text { value range }\end{array}$ \\
\hline$m_{v}$ & $0.552 \mathrm{~kg}$ \\
$W$ & $0.13 \mathrm{~m}$ \\
$L$ & $0.525 \mathrm{~m}$ \\
$l$ & $0.185 \mathrm{~m}-2.15 \mathrm{~m}$ \\
$H_{i}$ & $0.025 \mathrm{~m}-0.11 \mathrm{~m}$ \\
$\rho_{i}$ & $1000 \mathrm{kgm}^{-3}$ (water) \\
\hline
\end{tabular}

Values of the parameters used in Cooker (6), which are a guide to those used in this paper.

at that point can then be used to plot other features of the fluid such as the surface elevation $h^{(1)}(x, t)$. In the next section we investigate numerical solutions of the dispersion relation (3.6) and highlight the range of resonances which can occur in the system.

\section{Results}

In this section we examine the properties of the dispersion relation (3.6), and produce numerical solutions investigating these properties for various parameters. The experimental configuration of Cooker (6) is used as a guide for the parameter ranges used in the numerical results presented in this paper. In this study we assume the vessel dimensions and mass are the same as those as given in table 1, unless stated otherwise, and the other parameters are chosen to lie approximately in the ranges given in table 1 .

\subsection{The case $n=2$}

Before considering the general case of $n-1$ baffles, we first examine the case of $n=2$ fluid compartments (1 baffle), to highlight the different type of solutions which can arise from the 
dispersion relation $\Delta_{2}(\omega)=0$. In this case the dispersion relation reduces to

$$
\Delta_{2}=\sin \left[\alpha_{0}^{(1)}\right] \sin \left[\alpha_{0}^{(2)}\right] D_{2}(\omega)=0,
$$

where

$$
\begin{aligned}
& D_{2}(\omega)=\left(-\left(M g-m_{v} l \omega^{2}\right)+2 l \omega^{2} \rho_{1} H_{1} W \frac{\left(c_{0}^{(1)}\right)^{2}}{k_{0}^{(1)}} \tan \left[\alpha_{0}^{(1)}\right]+2 l \omega^{2} \rho_{2} H_{2} W \frac{\left(c_{0}^{(2)}\right)^{2}}{k_{0}^{(2)}} \tan \left[\alpha_{0}^{(2)}\right]\right. \\
& \left.+2 l \omega^{2} \rho_{1} H_{1} W \sum_{m=1}^{\infty} \frac{\left(c_{m}^{(1)}\right)^{2}}{k_{m}^{(1)}} \tanh \left[\alpha_{m}^{(1)}\right]+2 l \omega^{2} \rho_{2} H_{2} W \sum_{m=1}^{\infty} \frac{\left(c_{m}^{(2)}\right)^{2}}{k_{m}^{(2)}} \tanh \left[\alpha_{m}^{(2)}\right]\right) \cos \left[\alpha_{0}^{(1)}\right] \cos \left[\alpha_{0}^{(2)}\right] .
\end{aligned}
$$

Each of the three factors in $\Delta_{2}$ from (4.1) can be zero in their own right giving a simple root of the dispersion relation, and this depends upon the parameters $l, H_{1}, H_{2}, \rho_{1}, \rho_{2}, m_{v}, x_{1}$, $L$ and $W$. Even for the two compartment problem, the number of parameters is already large but their arbitrary choice allows the roots of $\Delta_{2}(\omega)=0$ to be chosen in different combinations.

\subsubsection{The lowest frequency solution}

For the 1 baffle problem, let us first consider the 3 situations

$$
\begin{array}{lll}
\sin \left[\alpha_{0}^{(1)}\right]=0, & \sin \left[\alpha_{0}^{(2)}\right] \neq 0, & D_{2}(\omega) \neq 0, \\
\sin \left[\alpha_{0}^{(1)}\right] \neq 0, & \sin \left[\alpha_{0}^{(2)}\right]=0, & D_{2}(\omega) \neq 0, \\
\sin \left[\alpha_{0}^{(1)}\right] \neq 0, & \sin \left[\alpha_{0}^{(2)}\right] \neq 0, & D_{2}(\omega)=0 .
\end{array}
$$

All three situations lead to simple roots of $\Delta_{2}(\omega)=0$, i.e. cases where $\Delta_{2}=0$, but $\Delta_{2}^{\prime} \neq 0$, and they correspond to non-resonant solutions to the sloshing problem. Although these solutions are non-resonant, they are still important, as the lowest frequency solution is likely to be the most commonly observed solution in an experiment.

The lowest frequency sloshing modes, with finite $l$, are always solutions of $D_{2}(\omega)=0$. To prove this, we assume w. l. o. g. that the product $\sin \left[\alpha_{0}^{(1)}\right] \sin \left[\alpha_{0}^{(2)}\right]=0$ first when $\alpha_{0}^{(1)}=\pi$ which we say occurs at $\omega=\omega_{1}$. Noting that $D_{2}(0)=-M g<0$, we see that at $\omega=\omega_{2}<\omega_{1}$, where $\alpha_{0}^{(1)}=\pi / 2$, then

$$
D_{2}\left(\omega_{2}\right)=2 l \omega_{2}^{2} \rho_{1} H_{1} W \frac{\left(c_{0}^{(1)}\right)^{2}}{k_{0}^{(1)}}>0,
$$

proving that there is always at least one root of $D_{2}(\omega)$ between $\omega=0$ and $\omega=\omega_{2}$ and thus there is always a root for $\omega<\omega_{1}$. The roots of the dispersion relation when $\sin \left[\alpha_{0}^{(i)}\right]=0$ are higher frequency solutions and correspond to symmetric sloshing modes which do not exert a force on the vessel, hence $\theta=0$ for these solutions.

The lowest frequency sloshing mode as a function of $H_{1}=H_{2}$ with $x_{1}=\frac{1}{2} L$ is plotted in figure 4 . The results show that the frequency of the sloshing mode increases rapidly for small fluid heights, and then increases slowly for larger fluid heights. This behaviour is in agreement 


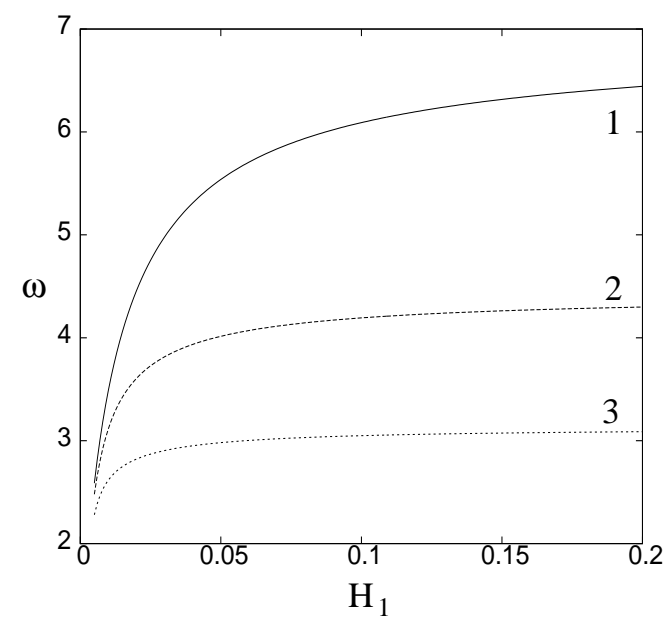

Fig. 4. Plot of $\omega\left(H_{1}\right)$ for the lowest frequency sloshing mode when $n=2, x_{1}=\frac{1}{2} L, H_{1}=H_{2}$ and $\rho_{1}=\rho_{2}$ for $l=0.2 \mathrm{~m}, 0.5 \mathrm{~m}$ and $1.0 \mathrm{~m}$ labelled $1-3$ respectively.

with the results presented in $\mathrm{Yu}(10)$ for the unbaffled problem. The difference here is that the baffle shortens each fluid compartment, so as $H_{1}$ increases the solution diverges from the shallow water approximation faster than for the unbaffled case.
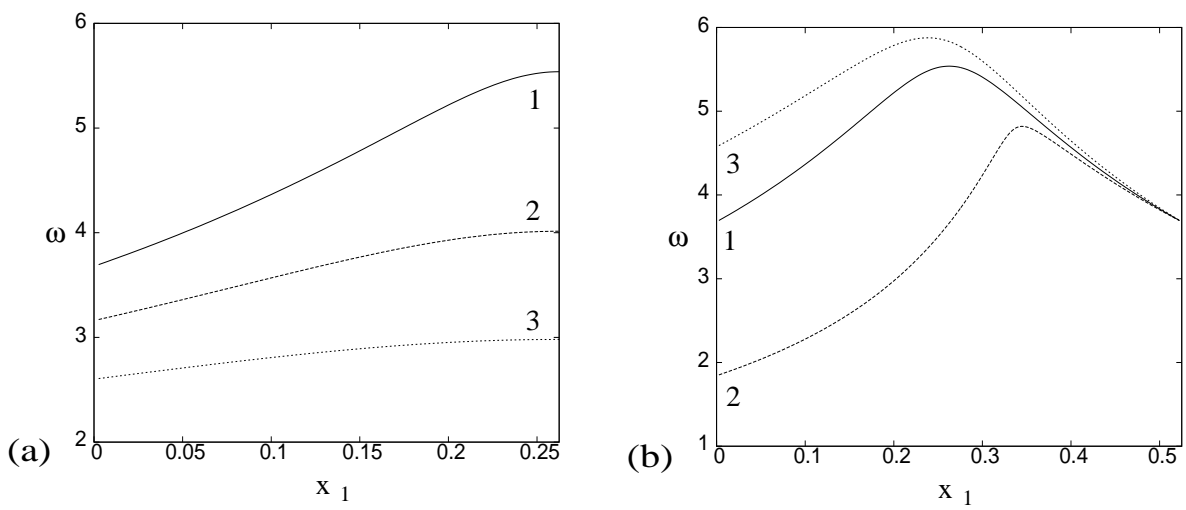

Fig. 5. Plot of (a) $\omega\left(x_{1}\right)$ for the lowest frequency sloshing mode when $n=2, H_{1}=H_{2}=0.05 \mathrm{~m}$ and $\rho_{1}=\rho_{2}$ for $l=0.2 \mathrm{~m}, 0.5 \mathrm{~m}$ and $1.0 \mathrm{~m}$ numbered $1-3$ respectively. Panel (b) shows $\omega\left(x_{1}\right)$ for the $l=0.2 \mathrm{~m}$ case in panel (a) (line 1) along with the results when $H_{2}=0.01 \mathrm{~m}$ (line 2) and $H_{2}=0.1 \mathrm{~m}$ (line 3).

If the height of the fluid in each compartment is now fixed such that $H_{1}=H_{2}=0.05 \mathrm{~m}$ and the position of the baffle, $x_{1}$, is varied, see figure $5(\mathrm{a})$, we observe that the frequency of the lowest sloshing mode increases from $x_{1}=0$ (effectively the unbaffled problem) to a maximum value when the baffle is in the centre of the vessel. The percentage change in the frequency of the lowest sloshing mode between $x_{1}=0$ and $x_{1}=\frac{1}{2} L$, increases as the string length is shortened, with the percentage increases in figure 5 (a) being $50 \%, 26 \%$ and $14 \%$ for results $1-3$ respectively.

Figure 5(b) shows the $l=0.2 \mathrm{~m}$ result from figure 5(a) (result 1 ) along with the corresponding result when the fluid height in compartment 2 is $H_{2}=0.01 \mathrm{~m}$ (line 2) and $H_{2}=0.1 \mathrm{~m}$ (line 3). This figure shows that the frequency of the lowest frequency sloshing mode is mainly dependent on the fluid in the longer of the two compartments, and all three results begin to agree for $x_{1} \gtrsim 0.325$ where $L_{1} \gtrsim \frac{3}{2} L_{2}$. If $H_{1}$ and $H_{2}$ are considerably different, then the compartment 

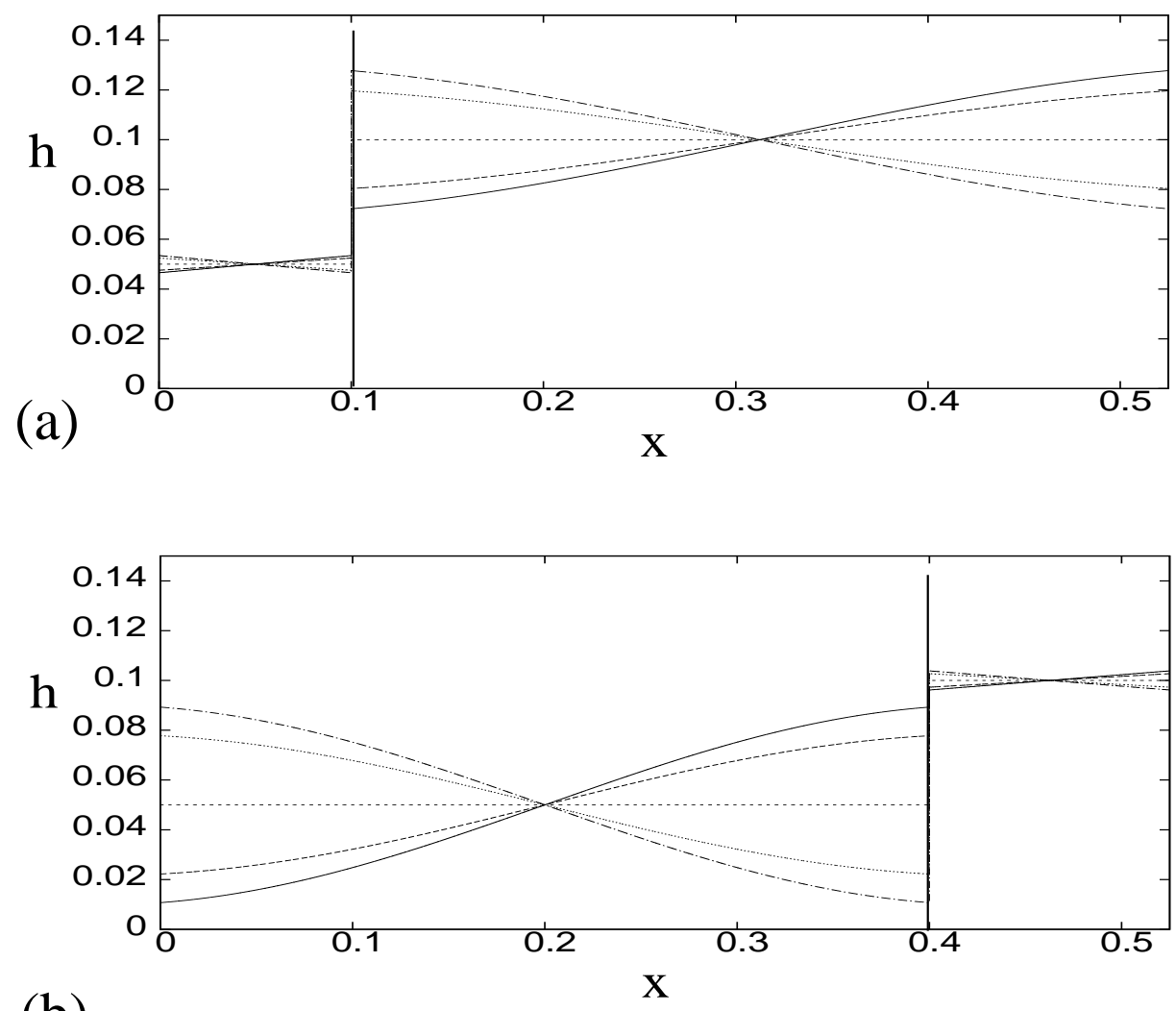

(b)

Fig. 6. Plot of the free surface elevation $h(x, t)$ for the lowest frequency sloshing mode when $H_{1}=0.05 \mathrm{~m}, H_{2}=0.1 \mathrm{~m}, \rho_{1}=\rho_{2}, l=0.2 \mathrm{~m}$ and (a) $x_{1}=0.1 \mathrm{~m}$ and (b) $x_{1}=0.4 \mathrm{~m}$. The elevation snapshots are taken at times $t=0$ (solid), $t=\pi /(4 \omega)$ (long dashed), $t=\pi /(2 \omega)$ (short dashed), $t=3 \pi /(4 \omega)$ (dotted) and $t=\pi / \omega$ (dot dashed). Here $\hat{\theta}=\pi / 50$.

lengths have to differ greatly for one fluid to dominate the sloshing frequency. However, in most practical situations, it is likely that the fluid heights in each compartment will be similar and so the vessel motion will depend upon the motion of both fluids. The free surface elevations for the case $H_{2}=0.1 \mathrm{~m}$ and $x_{1}=0.1 \mathrm{~m}$ and $x_{1}=0.4 \mathrm{~m}$ are plotted in figure 6 . Here we can see that the motion in the shorter compartment is much smaller than that in the longer compartment and so confirms that the vessel motion is being driven by the fluid motion in the larger of the two compartments.

\subsubsection{The $1: 1$ and $1: 1: 1$ resonances}

In the unbaffled case, an internal 1: 1 resonance was found by Alemi Ardakani et al. (11) when the frequency of one of the symmetric sloshing modes was equal to the frequency of the anti-symmetric modes and the vessel. These resonances can lead to energy pathways between modes in the nonlinear problem, and as such are very significant (19). These $1: 1$ resonances correspond to double roots of the dispersion relation $\Delta_{2}=0$, or solutions where $\Delta_{2}=\Delta_{2}^{\prime}=0$ with $\Delta_{2}^{\prime \prime} \neq 0$. The $1: 1$ resonances that occur in the two compartment problem are slightly different from the single compartment case, as there are two different types of $1: 1$ resonance. 
There are 3 different situations where a double zero occurs in the dispersion relation

$$
\begin{array}{lll}
\sin \left[\alpha_{0}^{(1)}\right]=0, & \sin \left[\alpha_{0}^{(2)}\right]=0, & D_{2}(\omega) \neq 0, \\
\sin \left[\alpha_{0}^{(1)}\right]=0, & \sin \left[\alpha_{0}^{(2)}\right] \neq 0, & D_{2}(\omega)=0, \\
\sin \left[\alpha_{0}^{(1)}\right] \neq 0, & \sin \left[\alpha_{0}^{(2)}\right]=0, & D_{2}(\omega)=0 .
\end{array}
$$

The first of these situations is a $1: 1$ resonance between the two fluids, which we call a 'nonvessel resonance', while the other two are resonances coupled to the vessel motion, and we call these 'vessel resonances'. The non-vessel resonance is just a free oscillation resonance in a stationary vessel, because in here the final row of (A.13) becomes

$$
\left(-\left(M g-m_{v} l \omega^{2}\right)+2 l \omega^{2} W \sum_{i=1}^{2} \rho_{i} H_{i} \sum_{m=1}^{\infty} \frac{\left(c_{m}^{(i)}\right)^{2}}{k_{m}^{(i)}} \tanh \left[\alpha_{m}^{(i)}\right]\right) \hat{\theta}=0,
$$

which implies that $\hat{\theta}=0$ if $D_{2}(\omega) \neq 0$ (The expression in the brackets is $D_{2}(\omega)$ with the two sine terms set to zero). In $\S 4.2$ we show that this is always the case, i.e. you cannot have a resonance between different fluid compartments unless the vessel is either stationary or unless the vessel is also in resonance with the fluid. The $1: 1$ resonance can never be the lowest frequency sloshing mode, due to the earlier argument (4.2), but it can occur as the second lowest frequency solution of the dispersion relation.

Figure 7 plots the value of $l\left(x_{1}\right)$ and $\omega\left(x_{1}\right)$ required to give a $1: 1$ vessel resonance in the two compartment system with the lowest frequency symmetric sloshing mode (i.e. when $\alpha_{0}^{(1)}$ or $\alpha_{0}^{(2)}=\pi$ ). When $H_{1}=H_{2}=0.05 \mathrm{~m}$ (panels (a) and (b)) the string length for a $1: 1$ resonance is around $1.2 \mathrm{~m}$ when compartment 1 is negligible in size $\left(x_{1} \approx 0\right)$. This is also the lowest possible resonance frequency, see panel (b). As the baffle position is moved across the vessel, the $1: 1$ resonance continues as a coupling with the rightmost compartment, but the string length required for resonance decreases down to zero at $x_{1} \approx 0.18 \mathrm{~m}$. There then exists a region where no $1: 1$ resonance occurs in the system with the lowest frequency symmetric sloshing mode, however it is possible that one exists for an integer multiple of $\pi$. When the baffle is placed at $x_{1} \approx 0.21 \mathrm{~m}$ the $1: 1$ resonance again emerges for very small values of $l$, but now the resonance occurs with the fluid in compartment 1, and the frequency of the sloshing mode is now the third lowest frequency solution of the dispersion relation. As the baffle is moved towards the centre of the vessel, there is a region where a $1: 1$ resonance can occur with either fluid compartment, depending on the length of the string. Long strings give a resonance with compartment 2 and short strings give a resonance with compartment 1 . At $x=\frac{1}{2} L$, the zeros of the dispersion relation combine giving a triple zero which corresponds to a $1: 1: 1$ resonance. This resonance is discussed in more detail later in this section.

The 1: 1 resonance results for $x_{1}>\frac{1}{2} L$ are the same as those for $x_{1}<\frac{1}{2} L$, except the compartments roles in the resonance are reversed due to the symmetry of this case. However, if $H_{1} \neq H_{2}$ then the symmetry of the solution is lost, but the overall structure of the $1: 1$ resonance remains, as can be seen in figures $7(\mathrm{c})$ and $7(\mathrm{~d})$.

The $1: 1: 1$ resonance in figure 7 occurs when

$$
\sin \left[\alpha_{0}^{(1)}\right]=\sin \left[\alpha_{0}^{(2)}\right]=D_{2}(\omega)=0,
$$



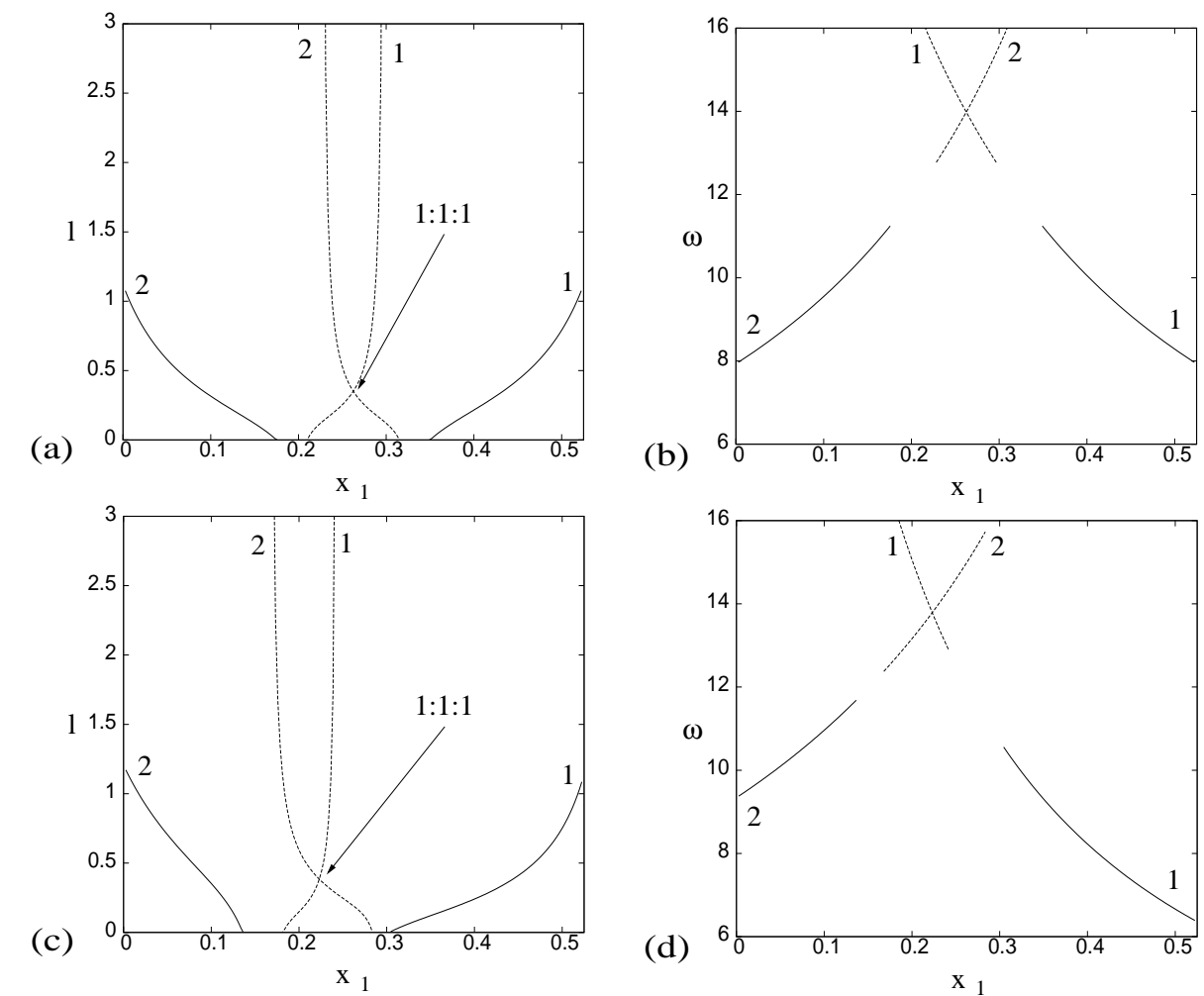

Fig. 7. Plot of (a,c) $l\left(x_{1}\right)$ and (b,d) $\omega\left(x_{1}\right)$ values which give the $1: 1$ resonance for $n=2$ with $\rho_{1}=\rho_{2}$ and $(\mathrm{a}, \mathrm{b})-H_{1}=H_{2}=0.05 \mathrm{~m}$ and $(\mathrm{c}, \mathrm{d})-H_{1}=0.03 \mathrm{~m}, H_{2}=0.08 \mathrm{~m}$. The labels 1 and 2 signify the compartment in which the symmetric fluid modes are resonating with the vessel, and the solid lines are when the $1: 1$ resonance is the second lowest frequency, and the dashed lines are when it is the third lowest frequency.

and corresponds to a solution of the dispersion relation where $\hat{\theta} \neq 0$. This resonance also corresponds to a solution where $\Delta_{2}=\Delta_{2}^{\prime}=\Delta_{2}^{\prime \prime}=0$ with $\Delta_{2}^{\prime \prime \prime} \neq 0$. In this case, both fluid motions and the vessel motion all have the same natural frequency. The $1: 1: 1$ resonance occurs at least as the third lowest frequency solution of the dispersion relation. This can be proved as follows. The 1:1:1 resonance must occur for the lowest natural frequency value where $\alpha_{0}^{(1)}=\alpha_{0}^{(2)}=\pi$ in (4.1). We say this occurs at $\omega=\omega_{1}$. At $\omega=\epsilon \ll 1, \Delta(\epsilon)<0$. At $\omega_{2}<\omega_{1}$ such that $\alpha_{0}^{(1)}=\alpha_{0}^{(2)}=\pi / 2, \Delta_{2}\left(\omega_{2}\right)=0$ as $D_{2}\left(\omega_{2}\right)=0$, but

$$
\begin{aligned}
\left.\frac{d \Delta_{2}}{d \omega}\right|_{\omega=\omega_{2}}=-2 l \omega_{2} W L_{2} \tanh ( & \left.\frac{\pi H_{1}}{L_{1}}\right)\left(\frac{\rho_{1} H_{1}\left(c_{0}^{(1)}\right)^{2} L_{2}}{L_{2} \tanh \left(\frac{\pi H_{2}}{L_{2}}\right)+\pi H_{2} \operatorname{sech}^{2}\left(\frac{\pi H_{2}}{L_{2}}\right)}\right. \\
& \left.+\frac{\rho_{2} H_{2}\left(c_{0}^{(2)}\right)^{2} L_{1}}{L_{1} \tanh \left(\frac{\pi H_{1}}{L_{1}}\right)+\pi H_{1} \operatorname{sech}^{2}\left(\frac{\pi H_{1}}{L_{1}}\right)}\right)<0 .
\end{aligned}
$$

Therefore, between $\omega=0$ and $\omega=\omega_{2}, \Delta_{2}$ must have a zero so it can pass through zero again at $\omega_{2}$ with a negative gradient. This shows that the solution at $\omega=\omega_{1}$ is at least the third lowest frequency solution. We observe however, that the root of $\Delta_{2}$ at $\omega=\omega_{2}$ when $\alpha_{0}^{(1)}=\alpha_{0}^{(2)}=\pi / 2$ 
(or odd integer multiples of $\pi / 2$ ) simplifies the matrix problem in (A.13) to

$$
\left[\begin{array}{ccc}
0 & 0 & l \omega_{2} c_{0}^{(1)} \\
0 & 0 & l \omega_{2} c_{0}^{(2)} \\
-W \rho_{1} c_{0}^{(1)} H_{1} \omega_{2} & -W \rho_{2} c_{0}^{(2)} H_{2} \omega_{2} & \sigma\left(\omega_{2}\right)
\end{array}\right]\left[\begin{array}{c}
\beta_{0}^{(1)} \\
\beta_{0}^{(2)} \\
\hat{\theta}
\end{array}\right]=\mathbf{0},
$$

which gives $\hat{\theta}=0$ and

$$
W \omega_{2}\left(\rho_{1} c_{0}^{(1)} H_{1} \beta_{0}^{(1)}+\rho_{2} c_{0}^{(2)} H_{2} \beta_{0}^{(2)}\right)=0 .
$$

Thus the signs of $\beta_{0}^{(1)}$ and $\beta_{0}^{(2)}$ are different and this gives free sloshing in a stationary vessel with both fluids out of phase with one another. This sloshing would be difficult to initiate in an experiment with $\theta \neq 0$ initially, unless there exists a heteroclinic orbit between the pure sloshing modes, as is the case in the unbaffled vessel (19).
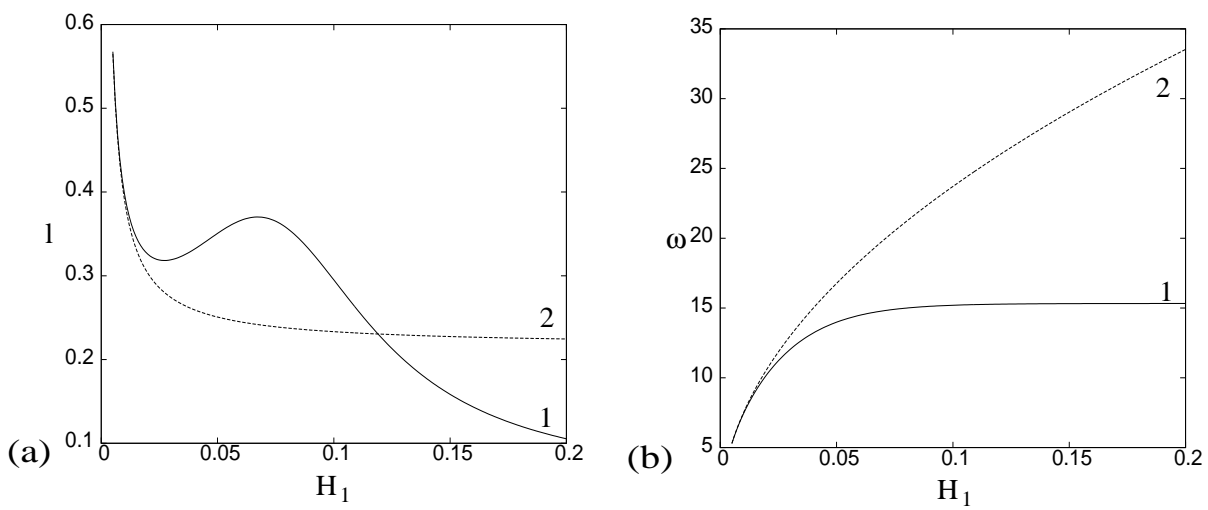

Fig. 8. Plot of (a) $l\left(H_{1}\right)$ which gives the $1: 1: 1$ resonance in the $n=2$ system with $x_{1}=\frac{1}{2} L$, $H_{2}=H_{1}$ and $\rho_{1}=\rho_{2}$ for the finite depth case (line 1) and the shallow water approximation (line 2). Panel (b) gives the corresponding frequency $\omega\left(H_{1}\right)$ at the $1: 1: 1$ resonance.

Figure 8(a) plots the value of $l$ as a function of $H_{1}=H_{2}$ which gives a $1: 1: 1$ resonance in a vessel with $x_{1}=\frac{1}{2} L$. The solid line gives the finite depth result, while the dashed line gives the shallow water approximation for the resonance (5.3), which is derived in $\S 5$. The finite depth result has a local maximum in $l$, followed by a decline in $l$ as $H_{1}$ is increased. This is compared to the shallow water approximation where $l$ decreases with $H_{1}$ with no local maximum. Figure 8(b) shows that the frequency of the $1: 1: 1$ resonance increases as $H_{1}$ is increased but at a much slower rate than the shallow water result. This variation in frequency is the same as was shown between the finite depth and shallow water results for the lowest frequency sloshing mode (10). If we assume that $l \approx 0.2 \mathrm{~m}$ is the minimum string length that can be achieved with the experimental setup from table 1 , then the $1: 1: 1$ resonance should be observable for values of $H_{1}=H_{2} \lesssim 0.12 \mathrm{~m}$. The resonance itself will not look unlike the lowest frequency solutions, as shown for the unbaffled case for Alemi Ardakani \& Brigdes (9). However, if a heteroclinic orbit exists for this system for a particular value of $H_{1}=H_{2}$ then the nonlinear response to the $1: 1: 1$ resonance should be observable. Note that if the vessel is set up with the parameters in figure 8 except with a different value of $l$, then the solution frequency would change and the solution would again be a non-resonant lowest frequency solution of $§ 4.1 .1$ if $\hat{\theta} \neq 0$ or a non-vessel resonance if $\theta=0$.

In figure 9 we plot the value of $l\left(x_{1}\right)$ which gives the $1: 1: 1$ resonance when $1-H_{1}=0.005 \mathrm{~m}$ and $2-H_{1}=0.5 \mathrm{~m}$. The corresponding value of $H_{2}$ comes from (A.8) by noting that $\omega^{2}$ is the 


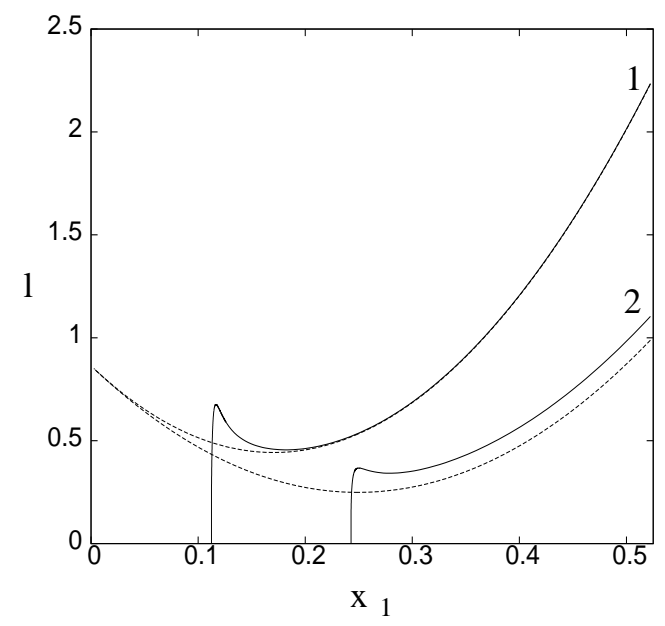

Fig. 9. Plot of $l\left(x_{1}\right)$ giving the $1: 1: 1$ resonance in the $n=2$ system when $\rho_{1}=\rho_{2}$ and 1$H_{1}=0.005 \mathrm{~m}$ and $2-H_{1}=0.05 \mathrm{~m}$ (solid lines). The value of $H_{2}$ comes from (4.3), and the dashed lines give the corresponding shallow water approximation.

same for both compartments, and so

$$
H_{2}=\frac{L_{2}}{2 \pi} \tanh ^{-1}\left(\frac{L_{2}}{L_{1}} \tanh \left(\frac{2 \pi H_{1}}{L_{1}}\right)\right)
$$

if $\alpha_{0}^{(1)}=\alpha_{0}^{(2)}=\pi$. The results in figure 9 show that when $x_{1} \approx L$, so that compartment 2 is negligible, there exists a value of $l$ for which the $1: 1: 1$ resonance exists. As $x_{1}$ is reduced, so that $L_{2}$ is allowed to increase in size, then $H_{2}$ increases and the value of $l$ decreases down to a minimum value. As $x_{1}$ is decreased further, there becomes a point where the value of $l$ begins to climb rapidly, which corresponds to $H_{2}$ becoming much larger, until eventually there is no solution to (4.3) and no $1: 1: 1$ resonance occurs. In the experimental setup, the value of $\mathrm{H}_{2}$ would be limited by the height of the vessel $d$, but unless the vessel is very shallow $d \ll L$, then this figure accurately depicts the experimental situation. This figure also shows that when $H_{1}$ is small, the shallow water approximation is in good agreement up to the point where the solution breaks down, but the shallow water approximation is not restricted by the break down of (4.3), and so the result of this approximation continues to $x_{1}=0$. However, as $H_{2}$ increases the shallow water approximation stops being valid and thus we expect results more like the finite depth results in this case.

It is worth noting here that in this experimental setup, we could also observe other resonances such as $1: 1: 2$ or $1: 2: 3$ for example, each of which is important in its own right. However, the focus of this paper is on highlighting the $(n+1)$-fold $1: 1: \cdots: 1$ resonance so we do not go into detail here, other than to acknowledge their existence.

The results from this section show that the inclusion of baffles into the vessel makes it more likely that a resonance of some kind $(1: 1$ or $1: 1: 1)$ will exist in experiments. If the number of baffles is increased further, this likelihood also increases. 


\subsection{General case of $n-1$ baffles}

For a general setup with a vessel containing $n$ distinct fluid compartments ( $n-1$ baffles), the number of possible resonances in the system increases to include $1: 1,1: 1: 1$ as well as multifold $1: \cdots: 1$ resonances up to and including the $(n+1)$-fold $1: 1: \cdots: 1$ resonance. As for the $n=2$ case, these resonances can be either a resonance between fluid compartments with the vessel stationary or a resonance between fluid motion and vessel motion.

A $p$-fold $1: 1: \cdots: 1$ resonance can occur in the system as one of ${ }^{n+1} C_{p}$ different resonant combinations, either as non-vessel resonances, or as one of ${ }^{n} C_{p-1}$ vessel resonances. Like the $n=2$ case, the case when $p=n$, such that all the $n$ fluid compartments are resonating together without coupling to the vessel, leads to a free oscillation resonance in a stationary vessel $(\hat{\theta}=0)$ from (A.13). The $p$-fold $1: 1: \cdots: 1$ resonance can also be considered as a solution to the problem when the first $p-1$ derivatives of the dispersion relation $\Delta_{n}(\omega)$ are zero and the $p^{\text {th }}$ derivative is non-zero.
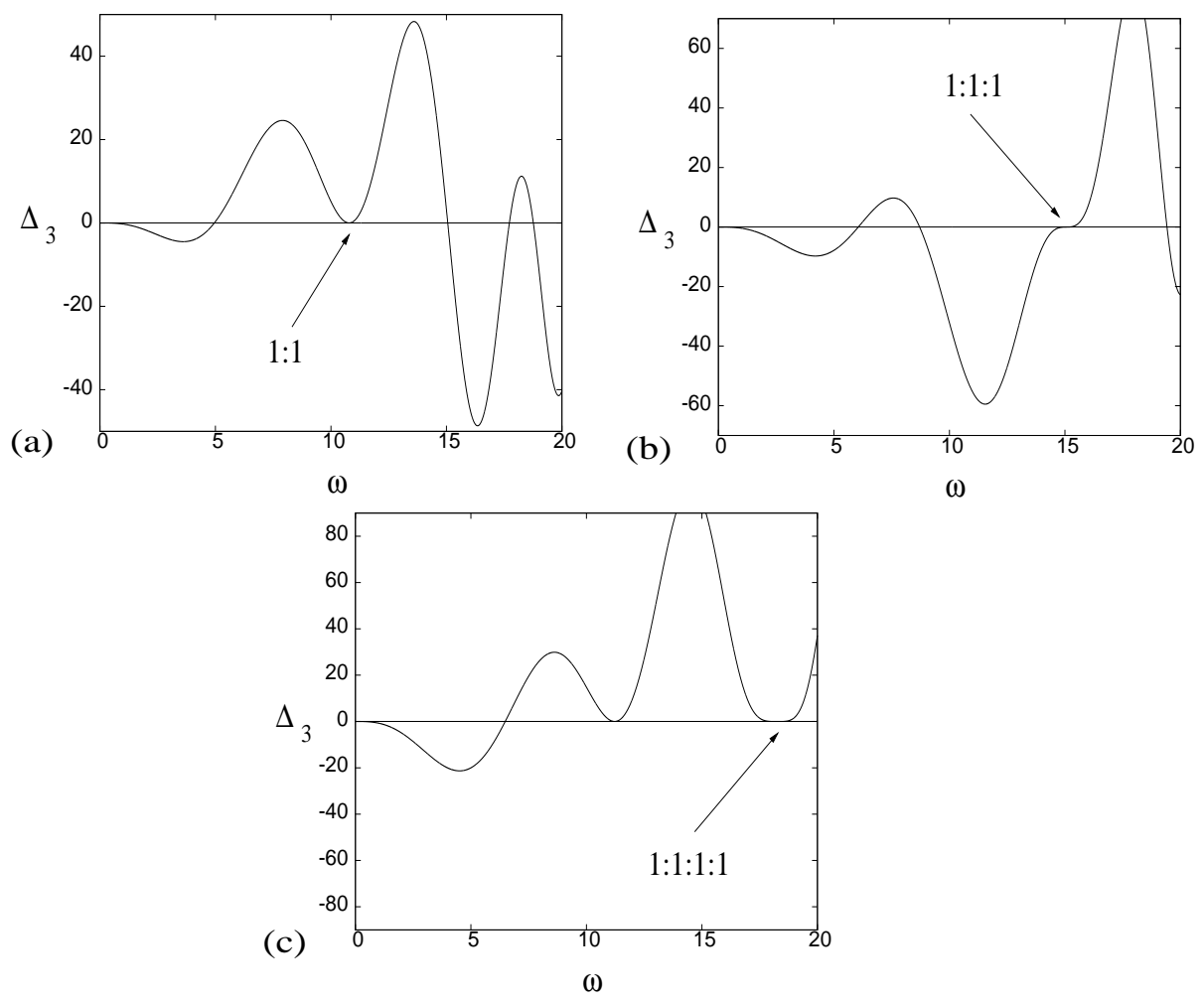

Fig. 10. Plot of $\Delta_{3}(\omega)$ with $\rho_{1}=\rho_{2}=\rho_{3}$ and $H_{1}=H_{2}=H_{3}=0.05 \mathrm{~m}$ showing (a) the $1: 1$ resonance as the second lowest frequency solution, (b) the $1: 1: 1$ resonance as the third lowest frequency solution and (c) the $1: 1: 1: 1$ resonance as the fourth lowest frequency solution of the dispersion relation. The compartment parameters are (a) $x_{1}=\frac{7}{10} L, x_{2}=\frac{9}{10} L, l=0.179 \mathrm{~m}$, (b) $x_{1}=\frac{45}{100} L$, $x_{2}=\frac{9}{10} L, l=0.176 \mathrm{~m}$ and (c) $x_{1}=\frac{1}{3} L, x_{2}=\frac{2}{3} L, l=0.182 \mathrm{~m}$.

In $\S 4.1$ we showed that for $n=2$ the $1: 1$ resonance occurs at least as the second lowest frequency solution of the dispersion relation, and the $1: 1: 1$ resonance is at least the third lowest. This structure generalises to the $n-1$ baffle problem, such that the $p$-fold $1: \cdots: 1$ resonance occurs at least as the $p^{\text {th }}$ lowest order frequency. However, we note that as for the $n=2$ case in figure 7 , there may be regions of parameter space where the $1: 1$ resonance 
cannot occur as the second lowest frequency etc. Figure 10 displays a set of examples for $n=3$ where the $1: 1,1: 1: 1$ and the $1: 1: 1: 1$ resonances occur as the second, third and fourth lowest frequencies respectfully. For the $1: 1: 1: 1$ resonance in figure 10(c), it should be noted that the solution at $\omega \approx 10$ is a double zero of the dispersion relation, and hence the quadruple zero at $\omega \approx 17$ is technically the fourth lowest frequency.

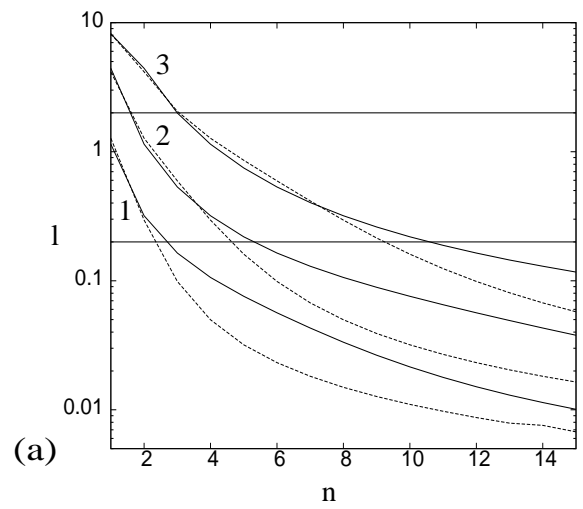

(b)

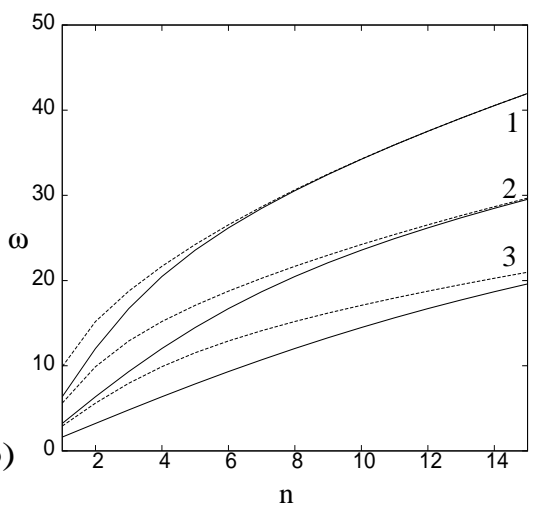

Fig. 11. Plot of (a) $l(n)$ and (b) $\omega(n)$ which gives the $(n+1)$-fold $1: \cdots: 1$ resonance with $x_{i}=i L / n$, $\rho_{i}=1000 \mathrm{kgm}^{-3}$ and $H_{i}=0.03 \mathrm{~m}$ (solid lines) and $H_{i}=0.1 \mathrm{~m}$ (dashed lines). The different sets of results are for $1-L=0.525 \mathrm{~m}, 2-L=1.05 \mathrm{~m}$ and $3-L=2.1 \mathrm{~m}$. The horizontal lines correspond to $l=0.2 \mathrm{~m}$ and $l=2 \mathrm{~m}$ which are the extremes of string length used by Cooker (6) in his experiment.

As the number of baffles in the vessel increases, the likelihood of observing interesting nonlinear behaviour close to a resonance in experiments increases, but the chances of observing the $(n+1)$-fold $1: \cdots: 1$ resonance reduces. This is because the string length required to achieve this resonance reduces rapidly with $n$, which leads to the frequency of the resonance also increasing with $n$. This can be seen in figure 11 for result 1 . Here, by $n=3$, the string length required to achieve the resonance reduces below $l=0.2 \mathrm{~m}$, which is approximately the minimum length used by Cooker in his experiment. However, increasing the length of the vessel by a factor of 2 (results 2) or 4 (results 3 ) shows that these higher order multifold resonances now lie in the string ranges used by Cooker. Here we have assumed that doubling the length of the vessel would also double its mass. Although for $L=1.05 \mathrm{~m}$ the $1: 1$ resonance with $n=1$ now lies outside the string range used by Cooker, it is now possible to observe the 7 -fold $1: \cdots: 1$ resonance from the $n=6$ case with $H_{i}=0.03 \mathrm{~m}$. For $L=2.1 \mathrm{~m}$ it might even be possible to see the $11-$ fold $1: \cdots: 1$ resonance in the $n=10$ case, but there will be other practical factors affecting this observation, such as finding strong enough cables to support the weight of the vessel and the fluid, but it is theoretically possible.

For the general case of $n-1$ baffles, if 2 compartments are resonating such that $\sin \left[\alpha_{0}^{(1)}\right]=$ $\sin \left[\alpha_{0}^{(2)}\right]=0$ (we can assume the resonance is between compartments 1 and 2 w. l. o. g.) then it can be shown that the system of equations (A.13) reduce to

$$
\begin{gathered}
\prod_{i=3}^{n} \sin \left[\alpha_{0}^{(i)}\right] \cos \left[\alpha_{0}^{(i)}\right]\left(-\left(M g-m_{v} l \omega^{2}\right)+2 l \omega^{2} W \sum_{i=3}^{n} \rho_{i} H_{i} \frac{\left(c_{0}^{(i)}\right)^{2}}{k_{0}^{(i)}} \tan \left[\alpha_{0}^{(i)}\right]\right. \\
\left.+2 l \omega^{2} W \sum_{i=1}^{n} \rho_{i} H_{i} \sum_{m=1}^{\infty} \frac{\left(c_{m}^{(i)}\right)^{2}}{k_{m}^{(i)}} \tanh \left[\alpha_{m}^{(i)}\right]\right) \hat{\theta}=0,
\end{gathered}
$$


once the $\beta_{0}^{(i)}$ 's have been eliminated which are given by

$$
k_{0}^{(i)} \beta_{0}^{(i)} \sin \left[2 \alpha_{0}^{(i)}\right]+l \omega c_{0}^{(i)}\left(1-\cos \left[2 \alpha_{0}^{(i)}\right]\right) \hat{\theta}=0,
$$

for $i=3, \ldots, n$. Therefore, for motions with $\hat{\theta} \neq 0$ this requires the term in brackets to be zero and the vessel is then resonating with the fluid compartments giving a $1: 1: 1$ resonance, otherwise these equations can only be satisfied with $\hat{\theta}=0$ and the resonance occurs as a free oscillation.

\section{Shallow water approximation}

The multi-fold resonances highlighted in $\S 3$ can also be found in the system when the shallow water approximation is taken in each compartment. Mathematically the shallow water limit is simple to determine and the governing dispersion relation for the solutions is given below. However, in practice this limit will be difficult to sustain for large values of $n$ as the compartment lengths will reduce for each additional baffle, unless the size of the vessel is increased.

For a given string length to vessel length ratio $l / L$, the shallow water approximation occurs when $k_{0}^{(i)} H_{i} \ll 1$ in each fluid compartment. In this limit the first equation of (A.8) gives

$$
\frac{\omega^{2} H_{i}}{g} \sim\left(k_{0}^{(i)} H_{i}\right)^{2}-\frac{1}{3}\left(k_{0}^{(i)} H_{i}\right)^{4}+O\left[\left(k_{0}^{(i)} H_{i}\right)^{6}\right]
$$

and by noting that each solution of the second equation of (A.8) lies in the range $(m-1) \pi<$ $k_{m}^{(i)} H_{i}<m \pi$ we can show that

$$
k_{m}^{(i)} H_{i} \sim m \pi-\frac{\left(k_{0}^{(i)} H_{i}\right)^{2}}{m \pi}-\left(\frac{1}{m^{2} \pi^{2}}-\frac{1}{3}\right) \frac{\left(k_{0}^{(i)} H_{i}\right)^{4}}{m \pi}+O\left[\left(k_{0}^{(i)} H_{i}\right)^{6}\right] .
$$

Substituting these expressions into (A.9) we find that

$$
\begin{aligned}
& c_{0}^{(i)}=1-\frac{1}{90}\left(k_{0}^{(i)} H_{i}\right)^{4}+O\left[\left(k_{0}^{(i)} H_{i}\right)^{6}\right], \\
& c_{m}^{(i)}=\frac{\sqrt{2}(-1)^{m+1}}{m^{2} \pi^{2}}\left(k_{0}^{(i)} H_{i}\right)^{2}+\frac{\sqrt{2}(-1)^{m}\left(2 m^{2} \pi^{2}-15\right)}{6 m^{4} \pi^{4}}\left(k_{0}^{(i)} H_{i}\right)^{4}+O\left[\left(k_{0}^{(i)} H_{i}\right)^{6}\right],
\end{aligned}
$$

and hence in this limit the evanescent modes become negligible in size compared to the wave mode. The dispersion relation (3.6) at leading order thus reduces to

$$
\Delta_{n}(\omega)^{\mathrm{SW}}=\left(-M g+m_{v} l \omega^{2}+l \omega^{2} \sum_{i=1}^{n} \frac{m_{f}^{(i)}}{\nu_{i}} \tan \left(\nu_{i}\right)\right) \prod_{i=1}^{n} \sin \left(\nu_{i}\right) \cos \left(\nu_{i}\right)
$$

where

$$
\nu_{i}=\lim _{k_{0}^{(i)} H_{i} \rightarrow 0} \alpha_{0}^{(i)}=\frac{L_{i} \omega}{2 \sqrt{g H_{i}}}
$$


The $(n+1)$-fold $1: 1: \cdots: 1$ resonance occurs at an $(n+1)$-fold root of $(5.1)$ which occurs precisely when

$$
\sin \left(\nu_{1}\right)=\ldots=\sin \left(\nu_{n}\right)=0 \quad \text { and } \quad \omega^{2}=\frac{M g}{m_{v} l},
$$

which is when the natural frequency of each compartment resonates with the vessel natural frequency. Assuming the solution in each compartment is the lowest frequency solution, then

$$
\nu_{i}=\pi \quad \forall i,
$$

and the system parameters are constrained by

$$
\frac{L_{i}}{\sqrt{l H_{i}}}=2 \pi \sqrt{\frac{m_{v}}{M}}
$$

This shallow water approximation is used in figures 8 and 9 of $\S 4$.

\section{Concluding Remarks}

This paper investigated the effect of non-porous baffles on the dynamic coupling between the sloshing fluid and the motion of the vessel in Cooker's sloshing experiment (6). It was found that the inclusion of $n-1$ baffles in the vessel, giving $n$ distinct fluid compartments, produced a number of different resonant behaviours, when the system parameters were tuned accordingly.

For a single baffle $(n=2)$ it was found that the $1: 1$ resonance of the single fluid compartment case (11) can also occur in this system. This occurs when the natural frequency of the antisymmetric fluid modes in one fluid compartment are tuned to the natural frequency of the vessel (vessel-resonance) or when both compartments contain symmetric sloshing modes with equal natural frequency (non-vessel resonance), with the vessel stationary. This system also included a $1: 1$ : 1 resonance, when the natural frequency of the anti-symmetric sloshing modes in both fluid compartments and the vessel were all equal. The inclusion of the baffle increased the region of parameter space for which a resonance can be observed when compared to the $n=1$, unbaffled case.

The general case with $n \geq 3$ was more interesting, because for particular system parameters, it contained any resonance from a $1: 1$ resonance through to an $(n+1)$-fold $1: 1: \cdots: 1$ resonance. The experimental set up used by Cooker (6) would contain the $1: 1: 1, n=2$ resonance, but by doubling the length, and mass, of the vessel, it would then contain resonances up to the 7 -fold $1: \cdots: 1$ resonance in the $n=6$ case.

The internal resonances highlighted in this paper are interesting, because they are precursors to dramatic dynamics when nonlinear effects are included in both the fluid and the vessel. Turner \& Bridges (19) showed that close to a $1: 1$ resonance in the unbaffled problem, the inclusion of nonlinear terms led to a pair of normal form equations

$$
\begin{aligned}
& \mathrm{i} a_{0} A_{\tau}=a_{1} \omega_{n, 2} A+a_{2}|A|^{2} A+a_{3}|B|^{2} A+a_{4} B^{2} A^{*}, \\
& \mathrm{i} b_{0} B_{\tau}=b_{1} \omega_{n, 2} B+b_{2}|B|^{2} B+a_{3}|A|^{2} B+a_{4} A^{2} B^{*}
\end{aligned}
$$

where $A(\tau), B(\tau)$ are the complex amplitudes of the linearly independent symmetric and anti-symmetric sloshing modes respectively, and the stars denote the complex conjugate. The 
parameters $a_{j}, b_{j}$ and $\omega_{n, 2}$ are all real and $\tau=\epsilon^{2} t$ with $\epsilon \ll 1$ is a slow time variable. This normal form has been investigated before in the literature in the context of three-dimensional rectangular vessels with almost square cross section (e.g. (18), (30)). A new analysis of this normal form was recently presented in Turner \& Bridges (19), obtained by projecting these equations onto a sphere, where all orbits become apparent. In the unbaffled case it was discovered that there are parameter values where a heteroclinic orbit exists which connects the purely symmetric sloshing modes with the purely anti-symmetric sloshing modes which allowed for complex motion in the system.

In the baffled system similar nonlinear normal forms would exist about each resonance, although they would be more complicated for higher order resonances. Effectively, a new complex amplitude and equation for each extra compartment would be needed. As the baffled problem contains more parameter regimes which contain resonances this means there are likely to be more fluid height combinations where there exist heteroclinic orbits between fluid modes and hence more chance of observing complex energy transfer between modes.

These nonlinear resonance effects could possibly be utilized in sea wave energy extraction devices in order to improve the efficiency with which they convert wave energy to electricity. The authors are currently involved in an EPSRC funded project modelling the flow inside the OWEL wave energy converter. This floating device consists of a rectangular compartment which is open at one end to allow the waves to enter, with the energy extracted via a power take-off system. The simplicity of the OWEL geometry makes the analysis of the current paper enlightening to future research avenues.

The final stages of this work was supported by the EPSRC under grant EP/K008188/1.

\section{A Derivation of the dispersion relation}

The system of linear equations (3.1)-(3.5) can be solved by seeking solutions in the form of time-periodic functions, with some undetermined frequency $\omega$. Thus we are searching for $\theta$, $\phi^{(i)}$ and $h_{i}$ in each fluid, of the form

$$
\phi^{(i)}(x, y, t)=\hat{\phi}^{(i)}(x, y) \cos \omega t, \quad h^{(i)}(x, t)=\hat{h}^{(i)}(x) \sin \omega t, \quad \theta(t)=\hat{\theta} \sin \omega t .
$$

From (3.2), the equation for the free surface $h^{(i)}$ in each fluid can be found via

$$
\hat{h}^{(i)}=\frac{\omega}{g} \hat{\phi}^{(i)}\left(x, H_{i}\right)
$$

thus $\hat{\theta}$ and $\hat{\phi}^{(i)}$ satisfy the boundary value problem

$$
\hat{\phi}_{x x}^{(i)}+\hat{\phi}_{y y}^{(i)}=0, \quad \text { in } \quad 0 \leq y \leq H_{i}, \quad x_{i-1} \leq x \leq x_{i},
$$

with the boundary equations 


$$
\begin{aligned}
& \hat{\phi}_{y}^{(i)}=0 \quad \text { on } \quad y=0, \\
& \hat{\phi}_{y}^{(i)}=\frac{\omega^{2}}{g} \hat{\phi}^{(i)} \quad \text { on } \quad y=H_{i}, \\
& \hat{\phi}_{x}^{(i)}=l \omega \hat{\theta} \quad \text { on } \quad x=x_{i-1}, x_{i} .
\end{aligned}
$$

These solutions in each compartment are coupled to the vessel motion via

$$
\left(M g-m_{v} l \omega^{2}\right) \hat{\theta}=W \omega \sum_{i=1}^{n} \int_{x_{i-1}}^{x_{i}} \int_{0}^{H_{i}} \rho_{i} \hat{\phi}_{x}^{(i)} d y d x
$$

The coupled set of equations (A.2)-(A.6) form an eigenvalue problem for the frequency $\omega$. The dispersion relation for this problem is found by solving (A.2) in each fluid as a function of $\hat{\theta}$, and then substituting these into (A.6) to derive an equation for the natural frequency $\omega$.

\section{A.1 The eigenmode expansion for $\hat{\phi}^{(i)}$}

The solution for each eigenmode $\hat{\phi}^{(i)}$ can be expressed in one of two ways:

(1) as an infinite sum of cosines $(16 ; 31)$, or

(2) as an infinites sum of vertical eigenmodes $(28 ; 10)$.

Alemi Ardakani et al. (11) showed that these two expressions are identical and so either could be used here. Turner \& Bridges (19) found that the vertical eigenmode expansion has numerical convergence problems close to the side walls of the vessel, which were significant for the solution of the nonlinear problem where these functions had to be integrated numerically. However, for the linear dispersion relation, no numerical integrations are required as the integrations can be evaluated exactly. Hence in this paper we use the vertical eigenmode expansion because it converges faster, and it simplifies in the shallow water limit, without the need to manipulate infinite series (12).

The eigenmode expansion used to solve the boundary value problem takes the form

$$
\hat{\phi}^{(i)}(x, y)=\sum_{m=0}^{\infty} A_{m}^{(i)}(x) \psi_{m}^{(i)}(y),
$$

where $\psi_{m}^{(i)}(y)$ are the complete set of vertical eigenmodes related to the vertical eigenvalue problem given in the appendix of Alemi Ardakani et al. (11) (see also (28)). The important details of these vertical eigenmodes are given in appendix B of Alemi Ardakani et al. (11), so we just quote the relevant results required for this paper. The vertical eigenmodes are ordered, such that the first eigenmode $(m=0)$ corresponds to the wave mode, which is the solitary vertical mode that exists in the shallow water limit, and the other modes are the evanescent modes, where this terminology comes from the wave-maker problem (see §2.2.1 of Linton \& McIver (28)). In the vertical eigenmode expansion, the symmetric and anti-symmetric sloshing modes are implicitly represented, however the resulting dispersion relation still has the same product form as (1.1). 
The solution of the eigenvalue problem in each compartment is identical, hence we only show it here for the $i^{\text {th }}$ compartment. By substituting (A.7) into (A.2) we determine that the functions $A_{m}^{(i)}(x)$ take the form

$$
\begin{aligned}
& A_{0}^{(i)}=\beta_{0}^{(i)} \cos \left[k_{0}^{(i)}\left(x-x_{i-1}\right)\right]+\gamma_{0}^{(i)} \sin \left[k_{0}^{(i)}\left(x-x_{i-1}\right)\right] \\
& A_{m}^{(i)}=\beta_{m}^{(i)} \cosh \left[k_{m}^{(i)}\left(x-x_{i-1}\right)\right]+\gamma_{m}^{(i)} \sinh \left[k_{m}^{(i)}\left(x-x_{i-1}\right)\right], \quad m=1,2, \ldots
\end{aligned}
$$

where $k_{0}^{(i)}$ and $k_{m}^{(i)}$ satisfy the relations

$$
\omega^{2}=g k_{0}^{(i)} \tanh \left(k_{0}^{(i)} H_{i}\right), \text { and } \omega^{2}=-g k_{m}^{(i)} \tan \left(k_{m}^{(i)} H_{i}\right), \text { for } m=1,2, \ldots .
$$

The boundary conditions at $x=x_{i-1}$ and $x_{i}$ reduce to

$$
\left.\frac{d A_{m}^{(i)}}{d x}\right|_{x=x_{i-1}}=\left.\frac{d A_{m}^{(i)}}{d x}\right|_{x=x_{i}}=l \omega \hat{\theta} c_{m}^{(i)}, \quad m=0,1, \ldots
$$

where the coefficients $c_{m}^{(i)}$ are given by

$$
c_{0}^{(i)}=\frac{1}{\sqrt{\frac{1}{2}\left(1+\frac{\sinh 2 k_{0}^{(i)} H_{i}}{2 k_{0}^{(i)} H_{i}}\right)}} \frac{\sinh k_{0}^{(i)} H_{i}}{k_{0}^{(i)} H_{i}}, \quad c_{m}^{(i)}=\frac{1}{\sqrt{\frac{1}{2}\left(1+\frac{\sin 2 k_{m}^{(i)} H_{i}}{2 k_{m}^{(i)} H_{i}}\right)}} \frac{\sin k_{m}^{(i)} H_{i}}{k_{m}^{(i)} H_{i}},
$$

which come from the expansion $1=\sum_{m=0}^{\infty} c_{m}^{(i)} \psi_{m}^{(i)}(y)$ in each compartment.

For the case $m=0$, evaluating the left wall boundary condition gives

$$
\gamma_{0}^{(i)}=\frac{l \omega \hat{\theta} c_{0}^{(i)}}{k_{0}^{(i)}}
$$

and thus the right wall boundary condition gives

$$
k_{0}^{(i)} \sin \left[2 \alpha_{0}^{(i)}\right] \beta_{0}^{(i)}+l \omega \hat{\theta} c_{0}^{(i)}\left(1-\cos \left[2 \alpha_{0}^{(i)}\right]\right)=0,
$$

where $\alpha_{m}^{(i)}=\frac{1}{2} k_{m}^{(i)} L_{i}$. At this stage it seems intuitive that we should divide by $\sin \left[\alpha_{0}^{(i)}\right]$ and write

$$
\beta_{0}^{(i)}=-\frac{l \omega \hat{\theta} c_{0}^{(i)}}{k_{0}^{(i)}} \tan \left[\alpha_{0}^{(i)}\right]
$$

However, $\sin \left[\alpha_{0}^{(i)}\right]$ could be zero, and retaining this term leads to a dispersion relation containing the internal resonances (11).

Applying the same solution method to the case when $m \geq 1$ leads to

$$
A_{m}^{(i)}=l \omega \hat{\theta} \frac{c_{m}^{(i)}}{k_{m}^{(i)}}\left(\sinh \left[k_{m}^{(i)}\left(x-x_{i-1}\right)\right]-\tanh \left[\alpha_{m}^{(i)}\right] \cosh \left[k_{m}^{(i)}\left(x-x_{i-1}\right)\right]\right) .
$$


However, this time we can divide through by $\sinh \left[\alpha_{m}^{(i)}\right]$ as it is non-zero for all $m$ and $i$ as we are only considering solutions with $\omega>0$. These evanescent modes are proportional to $\hat{\theta}$, and so clearly couple to the motion of the vessel.

\section{A.2 The dispersion relation}

The dispersion relation for the natural sloshing frequency of oscillation, $\omega$, is found by substituting the eigenmode expansion (A.7) into (A.6) and solving for $\omega$. In order to do this we need to determine the RHS of (A.6). We can again concentrate on the term for the $i^{\text {th }}$ fluid compartment as the result in each compartment is identical.

We note that

$$
\begin{aligned}
\int_{x_{i-1}}^{x_{i}} \int_{0}^{H_{i}} \rho_{i} \hat{\phi}_{x}^{(i)} d y d x & =\rho_{i} \int_{x_{i-1}}^{x_{i}} \int_{0}^{H_{i}} \sum_{m=0}^{\infty} \frac{d A_{m}^{(i)}}{d x}(x) \psi_{m}^{(i)}(y) d y d x \\
& =\rho_{i} H_{i} \sum_{m=0}^{\infty} c_{m}^{(i)}\left(A_{m}^{(i)}\left(x_{i}\right)-A_{m}^{(i)}\left(x_{i-1}\right)\right)
\end{aligned}
$$

using $\int_{0}^{H_{i}} \psi_{m}^{(i)} d y=H_{i} c_{m}^{(i)}$. Then by noting that

$$
\begin{aligned}
& A_{0}^{(i)}\left(x_{i}\right)-A_{0}^{(i)}\left(x_{i-1}\right)=\left(\cos \left[2 \alpha_{0}^{(i)}\right]-1\right) \beta_{0}^{(i)}+l \omega \hat{\theta} \frac{c_{0}^{(i)}}{k_{0}^{(i)}} \sin \left[2 \alpha_{0}^{(i)}\right], \\
& A_{m}^{(i)}\left(x_{i}\right)-A_{m}^{(i)}\left(x_{i-1}\right)=2 l \omega \hat{\theta} \frac{c_{m}^{(i)}}{k_{m}^{(i)}} \tanh \left[\alpha_{m}^{(i)}\right], \quad m \geq 1,
\end{aligned}
$$

it also becomes clear that

$$
\begin{array}{r}
\int_{x_{i-1}}^{x_{i}} \int_{0}^{H_{i}} \rho_{i} \hat{\phi}_{x}^{(i)} d y d x=\rho_{i} c_{0}^{(i)} H_{i} \beta_{0}^{(i)}\left(\cos \left[2 \alpha_{0}^{(i)}\right]-1\right) \\
+\rho_{i} H_{i} l \omega \hat{\theta} \frac{\left(c_{0}^{(i)}\right)^{2}}{k_{0}^{(i)}} \sin \left[2 \alpha_{0}^{(i)}\right]+2 \rho_{i} H_{i} l \omega \hat{\theta} \sum_{m=1}^{\infty} \frac{\left(c_{m}^{(i)}\right)^{2}}{k_{m}^{(i)}} \tanh \left[\alpha_{m}^{(i)}\right] .
\end{array}
$$

Summing over the $n$ fluid compartments gives the RHS of (A.6) as

$$
\begin{aligned}
& \sum_{i=1}^{n} \int_{x_{i-1}}^{x_{i}} \int_{0}^{H_{i}} \rho_{i} \hat{\phi}_{x}^{(i)} d y d x=\sum_{i=1}^{n} \rho_{i} c_{0}^{(i)} H_{i} \beta_{0}^{(i)}\left(\cos \left[2 \alpha_{0}^{(i)}\right]-1\right) \\
& +l \omega \hat{\theta} \sum_{i=1}^{n} \rho_{i} H_{i} \frac{\left(c_{0}^{(i)}\right)^{2}}{k_{0}^{(i)}} \sin \left[2 \alpha_{0}^{(i)}\right]+2 l \omega \hat{\theta} \sum_{i=1}^{n} \rho_{i} H_{i} \sum_{m=1}^{\infty} \frac{\left(c_{m}^{(i)}\right)^{2}}{k_{m}^{(i)}} \tanh \left[\alpha_{m}^{(i)}\right] .
\end{aligned}
$$

Therefore, the coupling equation with the vessel (A.6) becomes 


$$
\begin{aligned}
\left(M g-m_{v} l \omega^{2}\right) \hat{\theta}= & W \omega \sum_{i=1}^{n} \rho_{i} c_{0}^{(i)} H_{i} \beta_{0}^{(i)}\left(\cos \left[2 \alpha_{0}^{(i)}\right]-1\right)+l \omega^{2} \hat{\theta} W \sum_{i=1}^{n} \rho_{i} H_{i} \frac{\left(c_{0}^{(i)}\right)^{2}}{k_{0}^{(i)}} \sin \left[2 \alpha_{0}^{(i)}\right] \\
& +2 l \omega^{2} \hat{\theta} W \sum_{i=1}^{n} \rho_{i} H_{i} \sum_{m=1}^{\infty} \frac{\left(c_{m}^{(i)}\right)^{2}}{k_{m}^{(i)}} \tanh \left[\alpha_{m}^{(i)}\right] .
\end{aligned}
$$

The system of linear equations (A.10) for $i=1, \ldots, n$ and the coupled vessel equation (A.12) can be written in matrix form as

$$
\mathbf{B}(\omega) \mathbf{a}=\mathbf{0},
$$

where $\mathbf{a}=\left(\beta_{0}^{(1)}, \beta_{0}^{(2)}, \ldots, \beta_{0}^{(n)}, \hat{\theta}\right)$ is an $(n+1)$ vector. The $(n+1) \times(n+1)$ coefficient matrix $\mathbf{B}$ has the nice form that

where

$$
\mathbf{B}(\omega)=\left[\begin{array}{cc}
\boldsymbol{\delta}(\omega) & \boldsymbol{\xi}(\omega) \\
\boldsymbol{\eta}(\omega)^{T} & \sigma(\omega)
\end{array}\right]
$$

$$
\boldsymbol{\delta}(\omega)=\operatorname{diag}\left[k_{0}^{(1)} \sin \left[2 \alpha_{0}^{(1)}\right], \ldots k_{0}^{(n)} \sin \left[2 \alpha_{0}^{(n)}\right]\right],
$$

is an $n$ dimensional diagonal matrix, the vectors $\boldsymbol{\xi}(\omega)$ and $\boldsymbol{\eta}(\omega)$ have dimension $n$ and are expressed as

$$
\boldsymbol{\xi}=\left(\begin{array}{c}
l \omega c_{0}^{(1)}\left(1-\cos \left[2 \alpha_{0}^{(1)}\right]\right) \\
\vdots \\
l \omega c_{0}^{(n)}\left(1-\cos \left[2 \alpha_{0}^{(n)}\right]\right)
\end{array}\right), \boldsymbol{\eta}=\left(\begin{array}{c}
W \rho_{1} c_{0}^{(1)} H_{1} \omega\left(\cos \left[2 \alpha_{0}^{(1)}\right]-1\right) \\
\vdots \\
W \rho_{n} c_{0}^{(n)} H_{n} \omega\left(\cos \left[2 \alpha_{0}^{(n)}\right]-1\right)
\end{array}\right)
$$

and

$\sigma(\omega)=l \omega^{2} W \sum_{i=1}^{n} \rho_{i} H_{i} \frac{\left(c_{0}^{(i)}\right)^{2}}{k_{0}^{(i)}} \sin \left[2 \alpha_{0}^{(i)}\right]-\left(M g-m_{v} l \omega^{2}\right)+2 l \omega^{2} W \sum_{i=1}^{n} \rho_{i} H_{i} \sum_{m=1}^{\infty} \frac{\left(c_{m}^{(i)}\right)^{2}}{k_{m}^{(i)}} \tanh \left[\alpha_{m}^{(i)}\right]$.

A solution to the homogeneous system (A.13) exists if and only if the determinant of the matrix $\mathbf{B}$ is zero. Setting the determinant to zero gives the dispersion relation. Due to the form of the matrix $\mathbf{B}$ the dispersion relation can be written as $\Delta_{n}(\omega)=0$ with

$$
\Delta_{n}(\omega)=\operatorname{det}(\mathbf{B})=\operatorname{det}(\boldsymbol{\delta})\left(\sigma-\boldsymbol{\eta}^{T} \boldsymbol{\delta}^{-1} \boldsymbol{\xi}\right)
$$

which, after some algebra, can be shown to give the result in (3.6)

\section{References}

[1] H. N. Abramson, W. H. Chu, and L. R. Garza. "Liquid sloshing in spherical tanks." AIAA Journal 1(2), 384-389 (1963).

[2] H.N. Abramson. The Dynamic Behavior of Liquids in Moving Containers. (NASA SP-106, Washington D. C., 1966).

[3] R. A. Ibrahim. Liquid Sloshing Dynamics. (Cambridge University Press, Cambridge, 2005). 
[4] O. M. Faltinsen and A. N. Timokha. Sloshing. (Cambridge University Press, Cambridge, 2009).

[5] N. N. Moiseyev and V. V. Rumyantsev. Dynamic Stability of Bodies Containing Fluid. (Springer-Verlag, New York, 1968).

[6] M. J. Cooker. "Water waves in a suspended container." Wave Motion 20, 385-395 (1994).

[7] P. D Weidman. "Synchronous sloshing in free and suspended containers." APS Division of Fluid Dynamics, 47th Annual Meeting, Atlanta, GA 20-22 November, (1994).

[8] P. D Weidman. "Sloshing in suspended containers." APS Division of Fluid Dynamics, 58th Annual Meeting, Chicago, IL 20-22 November, (2005).

[9] H. Alemi Ardakani and T. J. Bridges. "Dynamic coupling between shallow-water sloshing and horizontal vehicle motion." Europ. J. Appl. Math 21, 479-517 (2010).

[10] J. Yu. "Effects of finite water depth on natural frequencies of suspended water tanks." Stud. Appl. Math. 125, 337-391 (2010).

[11] H. Alemi Ardakani, T. J. Bridges, and M. R. Turner. "Resonance in a model for Cooker's sloshing experiment." Euro. J. Mech. B/Fluids 36, 25-38 (2012).

[12] H. Alemi Ardakani, T. J. Bridges, and M. R. Turner. "Resonance in a model for Cooker's sloshing experiment - extended version." Technical Report, University of Surrey Repository (2012). http://epubs. surrey.ac.uk/713639/.

[13] Z. C. Feng and P. R. Sethna. "Global bifurcation and chaos in parametrically forced systems with 1: 1 resonance." Dynamical Systems: an Inter. Journal 5, 201-225 (1990).

[14] I. Hoveijn and O. N. Kirillov. "Singularities on the boundary of the stability domain near 1 : 1-resonance." J. Diff. Eqns. 248, 2585-2607 (2010).

[15] T. Ikeda and N. Nakagawa. "Non-linear vibrations of a structure caused by water sloshing in a rectangular tank." J. Sound Vibr. 201, 23-41 (1997).

[16] J. B. Frandsen. "Numerical predictions of tuned liquid tank structural systems." J. Fluids \& Structures 20, 309-329 (2005).

[17] T. Ikeda. "Autoparametric resonances in elastic structures carrying two rectangular tanks partially filled with liquid." J. Sound Vibr. 302, 657-682 (2007).

[18] Z. C. Feng and P. R. Sethna. "Symmetry breaking bifurcations in resonant surface waves." J. Fluid Mech. 199, 495-518 (1989).

[19] M. R. Turner and T. J. Bridges. "Nonlinear energy transfer between fluid sloshing and vessel dynamics." J. Fluid Mech. 719, 606-636 (2013).

[20] R. A. Struble and J. H. Heinbockel. "Resonant oscillations of a beam-pendulum system." J. Appl. Math. 30, 181-188 (1963).

[21] A. Skeldon and T. Mullin. "Mode interaction in a double pendulum." Phys. Lett. A 166, 224-229 (1992).

[22] R. Cushman. "Geometry of the bifurcations of the normalized reduced Hénon-Heiles family." Proc. Roy. Soc. London A 382, 361-371 (1982).

[23] K. Efstathiou, D. Sadovskii, and R. Cushman. "Linear Hamiltonian Hopf bifurcation for point-group-invariant perturbations of the $1: 1$ : 1 resonance." Proc. Roy. Soc. London A 459, 2997-3019 (2003).

[24] K. Efstathiou and D. Sadovskii. "Perturbations of the $1: 1: 1$ resonance with tetrahedral symmetry: a three degree of freedom analogue of the two degree of freedom Hénon-Heiles Hamiltonian." Nonlinearity 17, 415-446 (2004).

[25] K. Efstathiou, R. Cushman, and D. Sadovskii. "Hamiltonian Hopf bifurcation of the hydrogen atom in crossed fields." Physica D 250, 250-274 (2004).

[26] N. J. Fitch, C. A. Weidner, L. P. Parazzoli, H. R. Dullin, and H. J. Lewandowski. "Experimental demonstration of classical Hamiltonian monodromy in the $1: 1: 2$ resonant elastic pendulum." Phys. Rev. Lett. 103, 034301 (2009).

[27] O. Shoshani and O. Gottlieb. "Nonlinear dynamics and orbital stability of a wake oscillator model for self-excited vortex-induced vibration of a spherical pendulum." Proceedings of the 7th European Nonlinear Dynamics Conference, Rome, Italy (Eds: D. Bernardini, G. 
Rega and F. Romeo), (2011).

[28] C. M. Linton and P. McIver. Handbook of Mathematical Techniques for Wave-Structure Interaction. (Chapman \& Hall/CRC, Boca Raton, 2001).

[29] J. P. Boyd. Chebyshev and Fourier Spectral Methods. (Dover, New York, 2001).

[30] O. M. Faltinsen, O. F. Rognebakke, and A. N. Timokha. "Resonant three-dimensional nonlinear sloshing in a square-base basin." J. Fluid Mech. 487, 1-42 (2003).

[31] A. Herczyński and P. D. Weidman. "Experiments on the periodic oscillation of free containers driven by liquid sloshing." J. Fluid Mech. 693, 216-242 (2012). 